\title{
Port-Hamiltonian systems: network modeling and control of nonlinear physical systems*
}

\author{
A.J. van der Schaft ${ }^{\dagger}$
}

February 3, 2004

\begin{abstract}
It is shown how port-based modeling of lumped-parameter complex physical systems (multi-body systems, electrical circuits, electromechanical systems, ..) naturally leads to a geometrically defined class of systems, called port-Hamiltonian systems. These are Hamiltonian systems defined with respect to a power-conserving geometric structure capturing the basic interconnection laws, and a Hamiltonian function given by the total stored energy. The structural properties of port-Hamiltonian systems are discussed, in particular the existence of Casimir functions and its implications for stability and stabiliztion. Furthermore it is shown how passivity-based control results from interconnecting the plant port-Hamiltonian system with a controller port-Hamiltonian system, leading to a closed-loop port-Hamiltonian system. Finally, extensions to the distributed-parameter case are provided by formulating boundary control systems as infinite-dimensional portHamiltonian systems .
\end{abstract}

\section{Introduction}

Nonlinear systems and control theory has witnessed tremendous developments over the last three decades, see for example the textbooks $[22,36]$. Especially the introduction of geometric tools like Lie brackets of vector fields on manifolds has greatly advanced the theory, and has enabled the proper generalization of many fundamental concepts known for linear control systems to the nonlinear world. While the emphasis in the seventies and the eighties has been primarily on the structural analysis of smooth nonlinear dynamical control systems, in the nineties this has been combined with analytic techniques for stability, stabilization and robust control, leading e.g. to backstepping techniques and nonlinear $H_{\infty^{-}}$control. Moreover, in the last decade the theory of passive systems, and its implications for regulation and tracking, has undergone a remarkable revival. This last development was also spurred by work in robotics on the possibilities of shaping by feedback the physical energy in such a way that it can be used as a suitable Lyapunov function for the control purpose at hand, see e.g. the influential paper [59]. This has led to what is sometimes called passivity-based control, see e.g. [38].

In the present paper we want to further stress the importance of modeling for nonlinear control theory. Of course, this is well-known for (nonlinear) control applications, but in our opinion also the development of nonlinear control theory for physical systems should be

\footnotetext{
*to appear in Advanced Dynamics and Control of Structures, Eds. H. Irschik, K. Schlacher

${ }^{\dagger}$ Department of Applied Mathematics, University of Twente, PO Box 217, 7500 AE Enschede, The Netherlands
} 
integrated with a theoretical framework for modelling. We discuss how network modeling of (lumped-parameter) physical systems naturally leads to a geometrically defined class of systems, called port-Hamiltonian systems ${ }^{1}$. This provides a unified mathematical framework for the description of physical systems stemming from different physical domains, such as mechanical, electrical, thermal, as well as mixtures of them.

Historically, the Hamiltonian approach has its roots in analytical mechanics and starts from the principle of least action, via the Euler-Lagrange equations and the Legendre transform, towards the Hamiltonian equations of motion. On the other hand, the network approach stems from electrical engineering, and constitutes a cornerstone of systems theory. While most of the analysis of physical systems has been performed within the Lagrangian and Hamiltonian framework, the network modelling point of view is prevailing in modelling and simulation of (complex) physical systems. The framework of port-Hamiltonian systems combines both points of view, by associating with the interconnection structure ("generalized junction structure" in bond graph terminology) of the network model a geometric structure given by a Poisson structure, or more generally a Dirac structure. The Hamiltonian dynamics is then defined with respect to this Poisson (or Dirac) structure and the Hamiltonian given by the total stored energy, as well as the energy-dissipating elements and the ports of the system. This is discussed in Section 2 for the case of Poisson structures (no algebraic constraints), and in Section 4 for the general case of Dirac structures. Dirac structures encompass the "canonical" structures which are classically being used in the geometrization of mechanics, since they also allow to describe the geometric structure of systems with constraints as arising from the interconnection of sub-systems. Furthermore, Dirac structures allow to extend the Hamiltonian description of distributed-parameter systems to include variable boundary conditions, leading to distributed-parameter port-Hamiltonian systems with boundary ports. This will be briefly explained in Section 5 based on [54, 28, 29].

The structural properties of lumped-parameter port-Hamiltonian systems are investigated in Section 3 through geometric tools stemming from the theory of Hamiltonian systems. It is indicated how the interconnection of port-Hamiltonian systems again leads to a portHamiltonian system, and how this may be exploited for control and design. In particular, we investigate the existence of Casimir functions for the feedback interconnection of a plant port-Hamiltonian system and a controller port-Hamiltonian system, leading to a reduced port-Hamiltonian system on invariant manifolds with shaped energy. We thus provide an interpretation of passivity-based control from an interconnection point of view.

Acknowledgements This paper is based on joint work with several co-authors. In particular we thank Bernhard Maschke and Romeo Ortega for fruitful collaborations. Some of the material covered in this paper has appeared in [47].

\footnotetext{
${ }^{1}$ In previous publications we also used the terminology "Port-Controlled Hamiltonian (PCH) systems", or "generalized port-controlled Hamiltonian systems".
} 


\section{Port-Hamiltonian systems}

\subsection{From the Euler-Lagrange and Hamiltonian equations to port- Hamiltonian systems}

Let us briefly recall the standard Euler-Lagrange and Hamiltonian equations of motion. The standard Euler-Lagrange equations are given as

$$
\frac{d}{d t}\left(\frac{\partial L}{\partial \dot{q}}(q, \dot{q})\right)-\frac{\partial L}{\partial q}(q, \dot{q})=\tau
$$

where $q=\left(q_{1}, \ldots, q_{k}\right)^{T}$ are generalized configuration coordinates for the system with $k$ degrees of freedom, the Lagrangian $L$ equals the difference $K-P$ between kinetic energy $K$ and potential energy $P$, and $\tau=\left(\tau_{1}, \ldots, \tau_{k}\right)^{T}$ is the vector of generalized forces acting on the system. Furthermore, $\frac{\partial L}{\partial \dot{q}}$ denotes the column-vector of partial derivatives of $L(q, \dot{q})$ with respect to the generalized velocities $\dot{q}_{1}, \ldots, \dot{q}_{k}$, and similarly for $\frac{\partial L}{\partial q}$. In standard mechanical systems the kinetic energy $K$ is of the form

$$
K(q, \dot{q})=\frac{1}{2} \dot{q}^{T} M(q) \dot{q}
$$

where the $k \times k$ inertia (generalized mass) matrix $M(q)$ is symmetric and positive definite for all $q$. In this case the vector of generalized momenta $p=\left(p_{1}, \ldots, p_{k}\right)^{T}$, defined for any Lagrangian $L$ as $p=\frac{\partial L}{\partial \dot{q}}$, is simply given by

$$
p=M(q) \dot{q},
$$

and by defining the state vector $\left(q_{1}, \ldots, q_{k}, p_{1}, \ldots, p_{k}\right)^{T}$ the $k$ second-order equations (1) transform into $2 k$ first-order equations

$$
\begin{aligned}
& \dot{q}=\frac{\partial H}{\partial p}(q, p) \quad\left(=M^{-1}(q) p\right) \\
& \dot{p}=-\frac{\partial H}{\partial q}(q, p)+\tau
\end{aligned}
$$

where

$$
H(q, p)=\frac{1}{2} p^{T} M^{-1}(q) p+P(q) \quad\left(=\frac{1}{2} \dot{q}^{T} M(q) \dot{q}+P(q)\right)
$$

is the total energy of the system. The equations (4) are called the Hamiltonian equations of motion, and $H$ is called the Hamiltonian. The following energy balance immediately follows from (4):

$$
\frac{d}{d t} H=\frac{\partial^{T} H}{\partial q}(q, p) \dot{q}+\frac{\partial^{T} H}{\partial p}(q, p) \dot{p}=\frac{\partial^{T} H}{\partial p}(q, p) \tau=\dot{q}^{T} \tau,
$$

expressing that the increase in energy of the system is equal to the supplied work (conservation of energy).

If the Hamiltonian $H(q, p)$ is assumed to be the sum of a positive kinetic energy and a potential energy which is bounded from below, that is

$$
\begin{aligned}
H(q, p) & =\frac{1}{2} p^{T} M^{-1}(q) p+P(q) \\
M(q)=M^{T}(q) & >0, \quad \exists C>-\infty \quad \text { such that } P(q) \geq C .
\end{aligned}
$$


then it follows that (4) with inputs $u=\tau$ and outputs $y=\dot{q}$ is a passive (in fact, lossless) state space system with storage function $H(q, p)-C \geq 0$ (see e.g. [61, 20,47] for the general theory of passive and dissipative systems). Since the energy is only defined up to a constant, we may as well as take as potential energy the function $P(q)-C \geq 0$, in which case the total energy $H(q, p)$ becomes nonnegative and thus itself is the storage function.

System (4) is an example of a Hamiltonian system with collocated inputs and outputs, which more generally is given in the following form

$$
\begin{aligned}
& \dot{q}=\frac{\partial H}{\partial p}(q, p) \quad, \quad(q, p)=\left(q_{1}, \ldots, q_{k}, p_{1}, \ldots, p_{k}\right) \\
& \dot{p}=-\frac{\partial H}{\partial q}(q, p)+B(q) u, \quad u \in \mathbb{R}^{m}, \\
& y=B^{T}(q) \frac{\partial H}{\partial p}(q, p) \quad\left(=B^{T}(q) \dot{q}\right), \quad y \in \mathbb{R}^{m},
\end{aligned}
$$

Here $B(q)$ is the input force matrix, with $B(q) u$ denoting the generalized forces resulting from the control inputs $u \in \mathbb{R}^{m}$. The state space of (8) with local coordinates $(q, p)$ is usually called the phase space. In case $m<k$ we speak of an underactuated system. If $m=k$ and the matrix $B(q)$ is everywhere invertible, then the Hamiltonian system is called fully actuated.

Because of the form of the output equations $y=B^{T}(q) \dot{q}$ we again obtain the energy balance

$$
\frac{d H}{d t}(q(t), p(t))=u^{T}(t) y(t)
$$

Hence if $H$ is non-negative (or, bounded from below), any Hamiltonian system (8) is a lossless state space system. For a system-theoretic treatment of the Hamiltonian systems (8), especially if the output $y$ can be written as the time-derivative of a vector of generalized configuration coordinates, we refer to e.g. [8, 43, 44, 10, 36].

A major generalization of the class of Hamiltonian systems (8) is to consider systems which are described in local coordinates as

$$
\begin{array}{ll}
\dot{x}=J(x) \frac{\partial H}{\partial x}(x)+g(x) u, & x \in \mathcal{X}, u \in \mathbb{R}^{m} \\
y=g^{T}(x) \frac{\partial H}{\partial x}(x), & y \in \mathbb{R}^{m}
\end{array}
$$

Here $J(x)$ is an $n \times n$ matrix with entries depending smoothly on $x$, which is assumed to be skew-symmetric

$$
J(x)=-J^{T}(x),
$$

and $x=\left(x_{1}, \ldots, x_{n}\right)$ are local coordinates for an $n$-dimensional state space manifold $\mathcal{X}$. Because of (11) we easily recover the energy-balance $\frac{d H}{d t}(x(t))=u^{T}(t) y(t)$, showing that $(10)$ is lossless if $H \geq 0$. We call (10) with $J$ satisfying (11) a port-Hamiltonian system with structure matrix $J(x)$ and Hamiltonian $H([24,30,25])$. Note that (8) (and hence (4)) is a particular case of (10) with $x=(q, p)$, and $J(x)$ being given by the constant skew-symmetric matrix $J=\left[\begin{array}{cc}0 & I_{k} \\ -I_{k} & 0\end{array}\right]$, and $g(q, p)=\left[\begin{array}{c}0 \\ B(q)\end{array}\right]$.

As an important mathematical note, we remark that in many examples the structure matrix $J$ will satisfy the "integrability" conditions

$$
\sum_{l=1}^{n}\left[J_{l j}(x) \frac{\partial J_{i k}}{\partial x_{l}}(x)+J_{l i}(x) \frac{\partial J_{k j}}{\partial x_{l}}(x)+J_{l k}(x) \frac{\partial J_{j i}}{\partial x_{l}}(x)\right]=0, i, j, k=1, \ldots, n
$$


In this case we may find, by Darboux's theorem (see e.g. [60]) around any point $x_{0}$ where the rank of the matrix $J(x)$ is constant, local coordinates $\tilde{x}=(q, p, s)=$ $\left(q_{1}, \ldots, q_{k}, p_{1}, \ldots, p_{k}, s_{1}, \ldots s_{l}\right)$, with $2 k$ the rank of $J$ and $n=2 k+l$, such that $J$ in these coordinates takes the form

$$
J=\left[\begin{array}{ccc}
0 & I_{k} & 0 \\
-I_{k} & 0 & 0 \\
0 & 0 & 0
\end{array}\right]
$$

The coordinates $(q, p, s)$ are called canonical coordinates, and $J$ satisfying (11) and (12) is called a Poisson structure matrix. In such canonical coordinates the equations (10) take the form

$$
\begin{aligned}
\dot{q} & =\frac{\partial H}{\partial p}(q, p, s)+g_{q}(q, p, s) u \\
\dot{p} & =-\frac{\partial H}{\partial q}(q, p, s)+g_{p}(q, p, s) u \\
\dot{s} & =g_{s}(q, p, s) u \\
y & =g_{q}^{T}(q, p, s) \frac{\partial H}{\partial q}(q, p, s)+g_{p}^{T}(q, p, s) \frac{\partial H}{\partial p}(q, p, s)+g_{s}^{T}(q, p, s) \frac{\partial H}{\partial s}(q, p, s)
\end{aligned}
$$

which is, apart from the appearance of the variables $s$, very close to the standard Hamiltonian form (8). In particular, if $g_{s}=0$, then the variables $s$ are merely an additional set of constant parameters.

Although traditionally Hamiltonian systems arise from the Euler-Lagrange equations of motion (which are usually derived from variational principles) the point of departure for the theory of port-Hamiltonian systems is different. Indeed, port-Hamiltonian systems arise systematically from network models of physical systems. In network models of complex physical systems the overall system is seen as the interconnection of energy-storing elements via basic interconnection (balance) laws as Newton's third law or Kirchhoff's laws, as well as powerconserving elements like transformers, kinematic pairs and ideal constraints, together with energy-dissipating elements. The basic point of departure for the theory of port-Hamiltonian systems is to formalize the basic interconnection laws together with the power-conserving elements by a geometric structure, and to define the Hamiltonian as the total energy stored in the system. Indeed, for the (restricted) form of port-Hamiltonian systems given above the structure matrix $J(x)$ and the input matrix $g(x)$ may be directly associated with the network interconnection structure, while the Hamiltonian $H$ is just the sum of the energies of all the energy-storing elements; see our papers [30, 24, 32, 31, 50, 52, 27, 46, 58]. In particular, network models of complex physical systems formalized within the (generalized) bond graph language $([41,7])$ can be shown to immediately lead to port-Hamiltonian systems; see e.g. [19].

Example 2.1 (LCTG circuits). Consider a controlled LC-circuit (see Figure 1) consisting of two inductors with magnetic energies $H_{1}\left(\varphi_{1}\right), H_{2}\left(\varphi_{2}\right)\left(\varphi_{1}\right.$ and $\varphi_{2}$ being the magnetic flux linkages), and a capacitor with electric energy $H_{3}(Q)$ ( $Q$ being the charge). If the elements are linear then $H_{1}\left(\varphi_{1}\right)=\frac{1}{2 L_{1}} \varphi_{1}^{2}, H_{2}\left(\varphi_{2}\right)=\frac{1}{2 L_{2}} \varphi_{2}^{2}$ and $H_{3}(Q)=\frac{1}{2 C} Q^{2}$. Furthermore let $V=u$ denote a voltage source. Using Kirchhoff's laws one immediately arrives at the dynamical 


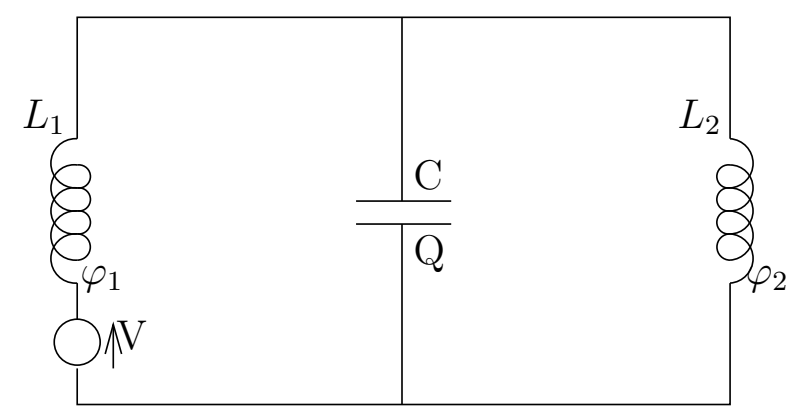

Figure 1: Controlled LC-circuit

equations

$$
\begin{aligned}
{\left[\begin{array}{c}
\dot{Q} \\
\dot{\varphi}_{1} \\
\dot{\varphi}_{2}
\end{array}\right]=\underbrace{\left[\begin{array}{ccc}
0 & 1 & -1 \\
-1 & 0 & 0 \\
1 & 0 & 0
\end{array}\right]}_{J}\left[\begin{array}{c}
\frac{\partial H}{\partial Q} \\
\frac{\partial H}{\partial \varphi_{1}} \\
\frac{\partial H}{\partial \varphi_{2}}
\end{array}\right]+\left[\begin{array}{l}
0 \\
1 \\
0
\end{array}\right] u } \\
y=\frac{\partial H}{\partial \varphi_{1}} \quad \text { (= current through first inductor) }
\end{aligned}
$$

with $H\left(Q, \varphi_{1}, \varphi_{2}\right):=H_{1}\left(\varphi_{1}\right)+H_{2}\left(\varphi_{2}\right)+H_{3}(Q)$ the total energy. Clearly the matrix $J$ is skewsymmetric, and since $J$ is constant it trivially satisfies (12). In [31] it has been shown that in this way every LC-circuit with independent elements can be modelled as a port-Hamiltonian system. Furthermore, also any LCTG-circuit with independent elements can be modelled as a port-Hamiltonian system, with $J$ determined by Kirchhoff's laws and the constitutive relations of the transformers $T$ and gyrators $G$.

Example 2.2 (Actuated rigid body). Consider a rigid body spinning around its center of mass in the absence of gravity. The energy variables are the three components of the body angular momentum $p$ along the three principal axes: $p=\left(p_{x}, p_{y}, p_{z}\right)$, and the energy is the kinetic energy

$$
H(p)=\frac{1}{2}\left(\frac{p_{x}^{2}}{I_{x}}+\frac{p_{y}^{2}}{I_{y}}+\frac{p_{z}^{2}}{I_{z}}\right),
$$

where $I_{x}, I_{y}, I_{z}$ are the principal moments of inertia. Euler's equations describing the dynamics are

$$
\left[\begin{array}{l}
\dot{p_{x}} \\
\dot{p_{y}} \\
\dot{p_{z}}
\end{array}\right]=\underbrace{\left[\begin{array}{ccc}
0 & -p_{z} & p_{y} \\
p_{z} & 0 & -p_{x} \\
-p_{y} & p_{x} & 0
\end{array}\right]}_{J(p)}\left[\begin{array}{l}
\frac{\partial H}{\partial p_{x}} \\
\frac{\partial H}{\partial p_{y}} \\
\frac{\partial H}{\partial p_{z}}
\end{array}\right]+g(p) u
$$

It can be checked that the skew-symmetric matrix $J(p)$ satisfies (12). (In fact, $J(p)$ is the canonical Lie-Poisson structure matrix on the dual of the Lie algebra so(3) corresponding to the configuration space $S O(3)$ of the rigid body.) In the scalar input case the term $g(p) u$ 
denotes the torque around an axis with coordinates $g=\left(\begin{array}{llll}b_{x} & b_{y} & b_{z}\end{array}\right)^{T}$, with corresponding collocated output given as

$$
y=b_{x} \frac{p_{x}}{I_{x}}+b_{y} \frac{p_{y}}{I_{y}}+b_{z} \frac{p_{z}}{I_{z}},
$$

which is the velocity around the same axis $\left(\begin{array}{lll}b_{x} & b_{y} & b_{z}\end{array}\right)^{T}$.

Example 2.3. A third important class of systems that naturally can be written as portHamiltonian systems, is constituted by mechanical systems with kinematic constraints. Consider as before a mechanical system with $k$ degrees of freedom, locally described by $k$ configuration variables $q=\left(q_{1}, \ldots, q_{k}\right)$. Suppose that there are constraints on the generalized velocities $\dot{q}$, described as

$$
A^{T}(q) \dot{q}=0,
$$

with $A(q)$ a $r \times k$ matrix of rank $r$ everywhere (that is, there are $r$ independent kinematic constraints). Classically, the constraints (18) are called holonomic if it is possible to find new configuration coordinates $\bar{q}=\left(\bar{q}_{1}, \ldots, \bar{q}_{k}\right)$ such that the constraints are equivalently expressed as

$$
\dot{\bar{q}}_{k-r+1}=\dot{\bar{q}}_{n-r+2}=\cdots=\dot{\bar{q}}_{k}=0,
$$

in which case one can eliminate the configuration variables $\bar{q}_{k-r+1}, \ldots, \bar{q}_{k}$, since the kinematic constraints (19) are equivalent to the geometric constraints

$$
\bar{q}_{k-r+1}=c_{k-r+1}, \ldots, \bar{q}_{k}=c_{k},
$$

for certain constants $c_{k-r+1}, \ldots, c_{k}$ determined by the initial conditions. Then the system reduces to an unconstrained system in the remaining configuration coordinates $\left(\bar{q}_{1}, \ldots, \bar{q}_{k-r}\right)$. If it is not possible to find coordinates $\bar{q}$ such that (19) holds (that is, if we are not able to integrate the kinematic constraints as above), then the constraints are called nonholonomic.

The equations of motion for the mechanical system with Lagrangian $L(q, \dot{q})$ and constraints (18) are given by the Euler-Lagrange equations [35]

$$
\begin{aligned}
\frac{d}{d t}\left(\frac{\partial L}{\partial \dot{q}}\right)-\frac{\partial L}{\partial q} & =A(q) \lambda+B(q) u, \quad \lambda \in \mathbb{R}^{r}, u \in \mathbb{R}^{m} \\
A^{T}(q) \dot{q} & =0
\end{aligned}
$$

where $B(q) u$ are the external forces (controls) applied to the system, for some $k \times m$ matrix $B(q)$, while $A(q) \lambda$ are the constraint forces. The Lagrange multipliers $\lambda(t)$ are uniquely determined by the requirement that the constraints $A^{T}(q(t)) \dot{q}(t)=0$ have to be satisfied for all $t$.

Defining as before (cf. (3)) the generalized momenta the constrained Euler-Lagrange equations (21) transform into constrained Hamiltonian equations (compare with (8)),

$$
\begin{aligned}
\dot{q} & =\frac{\partial H}{\partial p}(q, p) \\
\dot{p} & =-\frac{\partial H}{\partial q}(q, p)+A(q) \lambda+B(q) u \\
y & =B^{T}(q) \frac{\partial H}{\partial p}(q, p) \\
0 & =A^{T}(q) \frac{\partial H}{\partial p}(q, p)
\end{aligned}
$$


with $H(q, p)=\frac{1}{2} p^{T} M^{-1}(q) p+P(q)$ the total energy. The constrained state space is therefore given as the following subset of the phase space:

$$
\mathcal{X}_{c}=\left\{(q, p) \mid A^{T}(q) \frac{\partial H}{\partial p}(q, p)=0\right\}
$$

One way of proceeding with these equations is to eliminate the constraint forces, and to reduce the equations of motion to the constrained state space. In [49] it has been shown that this leads to a port-Hamiltonian system (10). Furthermore, the structure matrix $J_{c}$ of the portHamiltonian system satisfies the integrability conditions (12) if and only if the constraints (18) are holonomic. (In fact, if the constraints are holonomic then the coordinates $s$ as in (13) can be taken to be equal to the "integrated constraint functions" $\bar{q}_{k-r+1}, \ldots, \bar{q}_{k}$ of (20), and the matrix $g_{s}$ as in (14) is zero.)

An alternative way of approaching the system (22) is to formalize it directly as an implicit port-Hamiltonian system, as will be sketched in Section 4.

\subsection{Basic properties of port-Hamiltonian systems}

As allude to above, port-Hamiltonian systems naturally arise from a network modeling of physical systems without dissipative elements, see our papers $[24,30,25,32,31,26,50,48$, $52,27,46]$. Recall that a port-Hamiltonian system is defined by a state space manifold $\mathcal{X}$ endowed with a triple $(J, g, H)$. The pair $(J(x), g(x)), x \in \mathcal{X}$, captures the interconnection structure of the system, with $g(x)$ modeling in particular the ports of the system. This is very clear in Example 2.1, where the pair $(J(x), g(x))$ is determined by Kirchhoff's laws, the paradigmatic example of a power-conserving interconnection structure, but it naturally holds for other physical systems without dissipation as well. Independently from the interconnection structure, the function $H: \mathcal{X} \rightarrow \mathbb{R}$ defines the total stored energy of the system. Furthermore, port-Hamiltonian systems are intrinsically modular in the sense that a power-conserving interconnection of a number of port-Hamiltonian systems again defines a port-Hamiltonian system, with its overall interconnection structure determined by the interconnection structures of the composing individual systems together with their power-conserving interconnection, and the Hamiltonian just the sum of the individual Hamiltonians (see $[52,46,11])$.

As we have seen before, a basic property of port-Hamiltonian systems is the energybalancing property $\frac{d H}{d t}(x(t))=u^{T}(t) y(t)$. Physically this corresponds to the fact that the internal interconnection structure is power-conserving (because of skew-symmetry of $J(x)$ ), while $u$ and $y$ are the power-variables of the ports defined by $g(x)$, and thus $u^{T} y$ is the externally supplied power.

From the structure matrix $J(x)$ of a port-Hamiltonian system one can directly extract useful information about the dynamical properties of the system. Since the structure matrix is directly related to the modeling of the system (capturing the interconnection structure) this information usually has a direct physical interpretation.

A very important property which may be directly inferred from the structure matrix is the existence of dynamical invariants independent of the Hamiltonian $H$, called Casimir functions. Consider the set of p.d.e.'s

$$
\frac{\partial^{T} C}{\partial x}(x) J(x)=0, \quad x \in \mathcal{X}
$$


in the unknown (smooth) function $C: \mathcal{X} \rightarrow \mathbb{R}$. If (24) has a solution $C$ then it follows that the time-derivative of $C$ along the port-controlled Hamiltonian system (10) satisfies

$$
\begin{aligned}
\frac{d C}{d t} & =\frac{\partial^{T} C}{\partial x}(x) J(x) \frac{\partial H}{\partial x}(x)+\frac{\partial^{T} C}{\partial x}(x) g(x) u \\
& =\frac{\partial^{T} C}{\partial x}(x) g(x) u
\end{aligned}
$$

Hence, for the input $u=0$, or for arbitrary input functions if additionally $\frac{\partial^{T} C}{\partial x}(x) g(x)=0$, the function $C(x)$ remains constant along the trajectories of the port-Hamiltonian system, irrespective of the precise form of the Hamiltonian $H$. A function $C: \mathcal{X} \rightarrow \mathbb{R}$ satisfying (24) is called a Casimir function (of the structure matrix $J(x)$ ).

The existence of non-trivial solutions $C$ to (24) clearly assumes that rank $J(x)$ $<\operatorname{dim} \mathcal{X}$, but is also related to the integrability conditions (12). In fact, if canonical coordinates $(q, p, s)$ as in (13) have been found, then the Casimir functions are precisely all functions $C: \mathcal{X} \rightarrow \mathbb{R}$ depending only on the $s$-coordinates.

From (25) it follows that the level sets $L_{C}:=\{x \in \mathcal{X} \mid C(x)=c\}, c \in \mathbb{R}$, of a Casimir function $C$ are invariant sets for the autonomous Hamiltonian system $\dot{x}=J(x) \frac{\partial H}{\partial x}(x)$. Furthermore, the dynamics $\dot{x}=J(x) \frac{\partial H}{\partial x}(x)$ restricted to any level set $L_{C}$ is given as the reduced Hamiltonian dynamics

$$
\dot{x}_{C}=J_{C}\left(x_{C}\right) \frac{\partial H_{C}}{\partial x}\left(x_{C}\right)
$$

with $H_{C}$ and $J_{C}$ the restriction of $H$, respectively $J$, to $L_{C}$. More generally, if $C=\left(C_{1}, \ldots, C_{r}\right)$ are independent Casimir functions, then in any set of local coordinates $\left(z_{1}, \ldots, z_{l}, C_{1}, \ldots, C_{r}\right)$ for $\mathcal{X}$ the Hamiltonian dynamics $\dot{x}=J(x) \frac{\partial H}{\partial x}(x)$ takes the form

$$
\left[\begin{array}{c}
\dot{z} \\
\dot{C}
\end{array}\right]=\left[\begin{array}{cc}
\tilde{J}(z, C) & 0 \\
0 & 0
\end{array}\right]\left[\begin{array}{c}
\frac{\partial H}{\partial z} \\
\frac{\partial H}{\partial C}
\end{array}\right],
$$

leading to the reduced Hamiltonian dynamics

$$
\dot{z}=\tilde{J}(z, C=c) \frac{\partial H}{\partial z}
$$

on any multi-level set $\left\{x \in \mathcal{X} \mid\left(C_{1}(x), \ldots, C_{r}(x)\right)=c \in \mathbb{R}^{r}\right\}$.

The existence of Casimir functions has immediate consequences for stability analysis of (10) for $u=0$. Indeed, if $C_{1}, \cdots, C_{r}$ are Casimirs, then by (24) not only $\frac{d H}{d t}=0$ for $u=0$, but

$$
\frac{d}{d t}\left(H+H_{a}\left(C_{1}, \cdots, C_{r}\right)\right)(x(t))=0
$$

for any function $H_{a}: \mathbb{R}^{r} \rightarrow \mathbb{R}$. Hence, even if $H$ is not positive definite at an equilibrium $x^{*} \in \mathcal{X}$, then $H+H_{a}\left(C_{1}, \cdots, C_{r}\right)$ may be positive definite at $x^{*}$ by a proper choice of $H_{a}$, and thus may serve as a Lyapunov function. This method for stability analysis is called the Energy-Casimir method, see e.g. [23].

Example 2.4 (Example 2.1 continued). The quantity $\phi_{1}+\phi_{2}$ is a Casimir function. 
Example 2.5 (Example 2.2 continued). The quantity $\frac{1}{2} p_{x}^{2}+\frac{1}{2} p_{y}^{2}+\frac{1}{2} p_{z}^{2}$ (total angular momentum) is a Casimir function.

For a further discussion of the dynamical properties of Hamiltonian systems (especially if $J$ satisfies the integrability conditions (12)) we refer to the extensive literature on this topic, see e.g. $[1,23]$.

\subsection{Port-Hamiltonian systems with dissipation}

Energy-dissipation is included in the framework of port-Hamiltonian systems (10) by terminating some of the ports by resistive elements. Indeed, consider instead of $g(x) u$ in (10) a term

$$
\left[g(x) g_{R}(x)\right]\left[\begin{array}{c}
u \\
u_{R}
\end{array}\right]=g(x) u+g_{R}(x) u_{R}
$$

and extend correspondingly the output equations $y=g^{T}(x) \frac{\partial H}{\partial x}(x)$ to

$$
\left[\begin{array}{c}
y \\
y_{R}
\end{array}\right]=\left[\begin{array}{c}
g^{T}(x) \frac{\partial H}{\partial x}(x) \\
g_{R}^{T}(x) \frac{\partial H}{\partial x}(x)
\end{array}\right]
$$

Here $u_{R}, y_{R} \in \mathbb{R}^{m_{r}}$ denote the power variables at the ports which are terminated by static resistive elements

$$
u_{R}=-F\left(y_{R}\right)
$$

where the resistive characteristic $F: \mathbb{R}^{m_{r}} \rightarrow \mathbb{R}^{m_{r}}$ satisfies

$$
y_{R}^{T} F\left(y_{R}\right) \geq 0, \quad y_{R} \in \mathbb{R}^{m_{r}}
$$

(In many cases, $F$ will be derivable from a so-called Rayleigh dissipation function $R: \mathbb{R}^{m_{r}} \rightarrow \mathbb{R}$ in the sense that $F\left(y_{R}\right)=\frac{\partial R}{\partial y_{R}}\left(y_{R}\right)$.) In the sequel we concentrate on port-Hamiltonian systems with ports terminated by linear resistive elements

$$
u_{R}=-S y_{R}
$$

for some positive semi-definite symmetric matric $S=S^{T} \geq 0$. Substitution of (32) into (28) leads to a model of the form

$$
\begin{aligned}
& \dot{x}=[J(x)-R(x)] \frac{\partial H}{\partial x}(x)+g(x) u \\
& y=g^{T}(x) \frac{\partial H}{\partial x}(x)
\end{aligned}
$$

where $R(x):=g_{R}(x) S g_{R}^{T}(x)$ is a positive semi-definite symmetric matrix, depending smoothly on $x$. In this case the energy-balancing property (8) takes the form

$$
\begin{aligned}
\frac{d H}{d t}(x(t)) & =u^{T}(t) y(t)-\frac{\partial^{T} H}{\partial x}(x(t)) R(x(t)) \frac{\partial H}{\partial x}(x(t)) \\
& \leq u^{T}(t) y(t) .
\end{aligned}
$$

showing that a port-Hamiltonian system is passive if the Hamiltonian $H$ is bounded from below. We call (33) a port-Hamiltonian system with dissipation. Note that in this case two 
geometric structures play a role: the internal interconnection structure given by $J(x)$, and an additional resistive structure given by $R(x)$, which is determined by the port structure $g_{R}(x)$ and the linear constitutive relations $u_{R}=-S y_{R}$ of the resistive elements.

Regarding Casimir functions for a port-Hamiltonian system with dissipation (33) we consider functions $C: \mathcal{X} \rightarrow \mathbb{R}$ satisfying the set of p.d.e.'s

$$
\frac{\partial^{T} C}{\partial x}(x)[J(x)-R(x)]=0, \quad x \in \mathcal{X}
$$

implying that the time-derivative of $C$ along solutions of the system (33) for $u=0$ is zero (irrespective of the Hamiltonian $H$ ).

A stronger notion of Casimir functions is obtained by considering functions $C: \mathcal{X} \rightarrow \mathbb{R}$ which are Casimir functions for both geometric structures defined by $J(x)$ and $R(x)$, that is

$$
\begin{aligned}
& \frac{\partial^{T} C}{\partial x}(x) J(x)=0 \\
& \frac{\partial^{T} C}{\partial x}(x) R(x)=0
\end{aligned}
$$

If (36) holds for independent functions $C_{1}, \ldots, C_{r}$, then in any set of local coordinates $(z, C)=$ $\left(z_{1}, \ldots z_{l}, C_{1}, \ldots C_{r}\right)$ the dynamics (33) for $u=0$ takes the form

$$
\left[\begin{array}{c}
\dot{z} \\
\dot{C}
\end{array}\right]=\left(\left[\begin{array}{cc}
\tilde{J}(z, C) & 0 \\
0 & 0
\end{array}\right]-\left[\begin{array}{cc}
\tilde{R}(z, C) & 0 \\
0 & 0
\end{array}\right]\right)\left[\begin{array}{c}
\frac{\partial H}{\partial z} \\
\frac{\partial H}{\partial C}
\end{array}\right],
$$

which can be restricted on any multi-level set $\left\{x \in \mathcal{X} \mid\left(C_{1}(x), \ldots, C_{r}(x)\right)=c \in \mathbb{R}^{r}\right\}$ to

$$
\dot{z}=[\tilde{J}(z, C=c)-\tilde{R}(z, C=c)] \frac{\partial H}{\partial z}(z, C=c)
$$

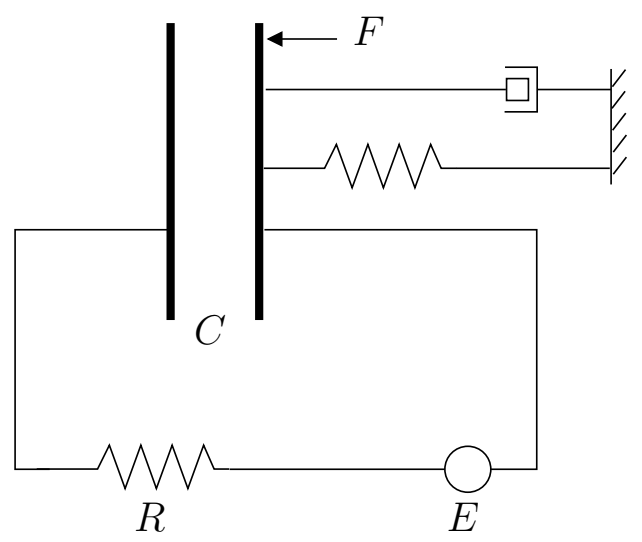

Figure 2: Capacitor microphone

Example 2.6. ([35]) Consider the capacitor microphone depicted in Figure 2. Here the capacitance $C(q)$ of the capacitor is varying as a function of the displacement $q$ of the right plate (with mass $m$ ), which is attached to a spring (with spring constant $k>0$ ) and a damper (with constant $c>0$ ), and affected by a mechanical force $F$ (air pressure arising from sound). 
Furthermore, $E$ is a voltage source. The dynamical equations of motion can be written as the port-Hamiltonian system with dissipation

$$
\begin{aligned}
& {\left[\begin{array}{c}
\dot{q} \\
\dot{p} \\
\dot{Q}
\end{array}\right]=\left(\left[\begin{array}{ccc}
0 & 1 & 0 \\
-1 & 0 & 0 \\
0 & 0 & 0
\end{array}\right]-\left[\begin{array}{ccc}
0 & 0 & 0 \\
0 & c & 0 \\
0 & 0 & 1 / R
\end{array}\right]\right)\left[\begin{array}{c}
\frac{\partial H}{\partial q} \\
\frac{\partial H}{\partial p} \\
\frac{\partial H}{\partial Q}
\end{array}\right]} \\
& +\left[\begin{array}{l}
0 \\
1 \\
0
\end{array}\right] F+\left[\begin{array}{c}
0 \\
0 \\
1 / R
\end{array}\right] E \\
& y_{1}=\frac{\partial H}{\partial p}=\dot{q} \\
& y_{2}=\frac{1}{R} \frac{\partial H}{\partial Q}=I
\end{aligned}
$$

with $p$ the momentum, $R$ the resistance of the resistor, $I$ the current through the voltage source, and the Hamiltonian $H$ being the total energy

$$
H(q, p, Q)=\frac{1}{2 m} p^{2}+\frac{1}{2} k(q-\bar{q})^{2}+\frac{1}{2 C(q)} Q^{2},
$$

with $\bar{q}$ denoting the equilibrium position of the spring. Note that $F \dot{q}$ is the mechanical power, and $E I$ the electrical power applied to the system. In the application as a microphone the voltage over the resistor will be used (after amplification) as a measure for the mechanical force $F$.

Example 2.7. ([Ortega et al. [39]]) A permanent magnet synchronous motor can be written as a port-Hamiltonian system with dissipation (in a rotating reference, i.e. the dq frame) for the state vector

$$
x=M\left[\begin{array}{c}
i_{d} \\
i_{q} \\
\omega
\end{array}\right], \quad M=\left[\begin{array}{ccc}
L_{d} & 0 & 0 \\
0 & L_{q} & 0 \\
0 & 0 & \frac{j}{n_{p}}
\end{array}\right]
$$

the magnetic flux linkages and mechanical momentum $\left(i_{d}, i_{q}\right.$ being the currents, and $\omega$ the angular velocity), $L_{d}, L_{q}$ stator inductances, $j$ the moment of inertia, and $n_{p}$ the number of pole pairs. The Hamiltonian $H(x)$ is given as $H(x)=\frac{1}{2} x^{T} M^{-1} x$ (total energy), while furthermore $J(x), R(x)$ and $g(x)$ are determined as

$$
\begin{aligned}
& J(x)=\left[\begin{array}{ccc}
0 & L_{0} x_{3} & 0 \\
-L_{0} x_{3} & 0 & -\Phi_{q 0} \\
0 & \Phi_{q 0} & 0
\end{array}\right], \\
& R(x)=\left[\begin{array}{ccc}
R_{S} & 0 & 0 \\
0 & R_{S} & 0 \\
0 & 0 & 0
\end{array}\right], g(x)=\left[\begin{array}{ccc}
1 & 0 & 0 \\
0 & 1 & 0 \\
0 & 0 & -\frac{1}{n_{p}}
\end{array}\right]
\end{aligned}
$$

with $R_{S}$ the stator winding resistance, $\Phi_{q 0}$ a constant term due to interaction of the permanent magnet and the magnetic material in the stator, and $L_{0}:=L_{d} n_{p} / j$. The three inputs are the stator voltage $\left(v_{d}, v_{q}\right)^{T}$ and the (constant) load torque. Outputs are $i_{d}, i_{q}$ and $\omega$. 
In some cases the interconnection structure $J(x)$ may be actually varying, depending on the mode of operation of the system, as exemplified by the following simple dc-to-dc power converter with a single switch. See for a further treatment of power converters in this context [39].

Example 2.8. Consider the ideal boost converter given in Figure 3.

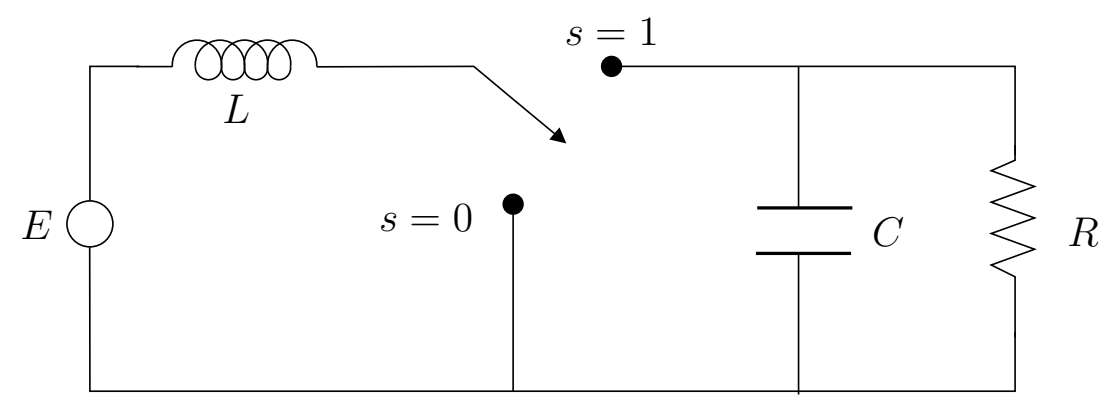

Figure 3: Ideal boost converter

The system equations are given as

$$
\begin{aligned}
{\left[\begin{array}{c}
\dot{x}_{1} \\
\dot{x}_{2}
\end{array}\right] } & =\left(\left[\begin{array}{cc}
0 & -s \\
s & 0
\end{array}\right]-\left[\begin{array}{cc}
0 & 0 \\
0 & 1 / R
\end{array}\right]\right)\left[\begin{array}{l}
\frac{\partial H}{\partial x_{1}} \\
\frac{\partial H}{\partial x_{2}}
\end{array}\right]+\left[\begin{array}{l}
1 \\
0
\end{array}\right] E \\
y & =\frac{\partial H}{\partial x_{1}}
\end{aligned}
$$

with $x_{1}$ the magnetic flux linkage of the inductor, $x_{2}$ the charge of the capacitor, and $H\left(x_{1}, x_{2}\right)=\frac{1}{2 L} x_{1}^{2}+\frac{1}{2 C} x_{2}^{2}$ the total stored energy. The internal interconnection structure matrix $J$ is either $\left[\begin{array}{ll}0 & 0 \\ 0 & 0\end{array}\right]$ or $\left[\begin{array}{cc}0 & -1 \\ 1 & 0\end{array}\right]$, depending on the ideal switch being in position $s=0$ or $s=1$.

\section{Control of port-Hamiltonian systems}

The aim of this section is to discuss a general methodology for port-Hamiltonian systems (with or without dissipation) which exploits their Hamiltonian properties in an intrinsic way, see e.g. [33, 34, 39, 47]. An expected benefit of such a methodology is that it leads to physically interpretable controllers, which possess inherent robustness properties. Future research is aimed at corroborating these claims.

We have already seen that port-Hamiltonian systems are passive if the Hamiltonian $H$ is bounded from below. Hence in this case we can use all the results from the theory of passive systems, such as asymptotic stabilization by the insertion of damping by negative output feedback, see e.g. [47]. The emphasis in this section is however on the somewhat complementary aspect of shaping the energy of the system, which directly involves the Hamiltonian structure of the system, as opposed to the more general passivity structure. 


\subsection{Control by interconnection}

Consider a port-Hamiltonian system (33)

$$
\begin{aligned}
\dot{x} & =[J(x)-R(x)] \frac{\partial H}{\partial x}(x)+g(x) u \\
y & =g^{T}(x) \frac{\partial H}{\partial x}(x)
\end{aligned} \quad x \in \mathcal{X}
$$

regarded as a plant system to be controlled. In the previous section we have seen that many physical systems can be modelled in this way, and that the defining entities $J(x), g(x), R(x)$ and $H$ have a concrete physical interpretation. Furthermore, if $H$ is bounded from below then (44) is a passive system.

Recall the well-known result that the standard feedback interconnection of two passive systems again is a passive system; a basic fact which can be used for various stability and control purposes. In the same vein we can consider the interconnection of the plant (44) with another port-Hamiltonian system

$$
C: \begin{aligned}
& \dot{\xi}=\left[J_{C}(\xi)-R_{C}(\xi)\right] \frac{\partial H_{C}}{\partial \xi}(\xi)+g_{C}(\xi) u_{C} \\
& y_{C}=g_{C}^{T}(\xi) \frac{\partial H_{C}}{\partial \xi}(\xi)
\end{aligned} \xi \in \mathcal{X}_{C}
$$

regarded as the controller system, via the standard feedback interconnection

$$
\begin{aligned}
& u=-y_{C}+e \\
& u_{C}=y+e_{C}
\end{aligned}
$$

with $e, e_{C}$ external signals inserted in the feedback loop. The closed-loop system takes the form

$$
\begin{aligned}
& {\left[\begin{array}{c}
\dot{x} \\
\dot{\xi}
\end{array}\right]=(\underbrace{\left[\begin{array}{cc}
J(x) & -g(x) g_{C}^{T}(\xi) \\
g_{C}(\xi) g^{T}(x) & J_{C}(\xi)
\end{array}\right]}_{J_{c l}(x, \xi)}-\underbrace{\left[\begin{array}{cc}
R(x) & 0 \\
0 & R_{C}(\xi)
\end{array}\right]}_{R_{c l}(x, \xi)})} \\
& \begin{array}{r}
{\left[\begin{array}{c}
\frac{\partial H}{\partial x}(x) \\
\frac{\partial H_{C}}{\partial \xi}(\xi)
\end{array}\right]+\left[\begin{array}{cc}
g(x) & 0 \\
0 & g_{C}(\xi)
\end{array}\right]} \\
{\left[\begin{array}{c}
y \\
y_{C}
\end{array}\right]=\left[\begin{array}{cc}
g(x) & 0 \\
0 & g_{C}(\xi)
\end{array}\right]\left[\begin{array}{c}
\frac{\partial H}{\partial x}(x) \\
\frac{\partial H_{C}}{\partial \xi}(\xi)
\end{array}\right]}
\end{array}
\end{aligned}
$$

which again is a port-Hamiltonian system, with state space given by the product space $\mathcal{X} \times \mathcal{X}_{C}$, total Hamiltonian $H(x)+H_{C}(\xi)$, inputs $\left(e, e_{C}\right)$ and outputs $\left(y, y_{C}\right)$. Hence the feedback interconnection of any two port-Hamiltonian systems results in another port-Hamiltonian system; just as in the case of passivity.

It is of interest to investigate the Casimir functions of the closed-loop system, especially those relating the state variables $\xi$ of the controller system to the state variables $x$ of the plant system. Indeed, from a control point of view the Hamiltonian $H$ is given while $H_{C}$ can be assigned. Thus if we can find Casimir functions $C_{i}(\xi, x), i=1, \cdots, r$, relating $\xi$ to $x$ then by the Energy-Casimir method the Hamiltonian $H+H_{C}$ of the closed-loop system may be replaced by the Hamiltonian $H+H_{C}+H_{a}\left(C_{1}, \cdots, C_{r}\right)$, thus creating the possibility of 
obtaining a suitable Lyapunov function for the closed-loop system.

In particular, let us consider Casimir functions of the form

$$
\xi_{i}-G_{i}(x) \quad, \quad i=1, \ldots, \operatorname{dim} \mathcal{X}_{C}=n_{C}
$$

That means (see (35)) that we are looking for solutions of the p.d.e.'s (with $e_{i}$ denoting the $i$-th basis vector)

$$
\left[\begin{array}{ll}
-\frac{\partial^{T} G_{i}}{\partial x}(x) & e_{i}^{T}
\end{array}\right]\left[\begin{array}{cc}
J(x)-R(x) & -g(x) g_{C}^{T}(\xi) \\
g_{C}(\xi) g^{T}(x) & J_{C}(\xi)-R_{C}(\xi)
\end{array}\right]=0
$$

or written out

$$
\begin{gathered}
\frac{\partial^{T} G_{i}}{\partial x}(x)[J(x)-R(x)]-g_{C}^{i}(\xi) g^{T}(x)=0 \\
\frac{\partial^{T} G_{i}}{\partial x}(x) g(x) g_{C}^{T}(\xi)+J_{C}^{i}(\xi)-R_{C}^{i}(\xi)=0
\end{gathered}
$$

with $\frac{\partial^{T} G_{i}}{\partial x}$ denoting as before the gradient vector $\left(\frac{\partial G_{i}}{\partial x_{1}}, \ldots, \frac{\partial G_{i}}{\partial x_{n}}\right)$, and $g_{C}^{i}, J_{C}^{i}, R_{C}^{i}$ denoting the i-th row of $g_{C}, J_{C}$, respectively $R_{C}$.

Suppose we want to solve (49) for $i=1, \ldots, \bar{n}$, with $\bar{n} \leq n_{C}$ (possibly after permutation of $\left.\xi_{1}, \ldots, \xi_{n_{C}}\right)$. with $\bar{J}_{C}(\xi), \bar{R}_{C}(\xi)$ the $\bar{n} \times \bar{n}$ left-upper submatrices of $J_{C}$, respectively $R_{C}$. The following proposition has been shown in [47].

Proposition 3.1. The functions $\xi_{i}-G_{i}(x), i=1, \ldots, \bar{n} \leq n_{C}$, satisfy (49) (and thus are Casimirs of the closed-loop port-controlled Hamiltonian system (47) for $e=0, e_{c}=0$ ) if and only if $G=\left(G_{1}, \ldots, G_{\bar{n}}\right)^{T}$ satisfies

$$
\begin{aligned}
& \frac{\partial^{T} G}{\partial x}(x) J(x) \frac{\partial G}{\partial x}(x)=\bar{J}_{C}(\xi) \\
& R(x) \frac{\partial G}{\partial x}(x)=0 \\
& \bar{R}_{C}(\xi)=0 \\
& \frac{\partial^{T} G}{\partial x}(x) J(x)=\tilde{g}_{C}(\xi) g^{T}(x)
\end{aligned}
$$

with $\bar{J}_{C}(\xi), \bar{R}_{C}(\xi)$ the $\bar{n} \times \bar{n}$ left-upper submatrices of $J_{C}$, respectively $R_{C}$.

In particular, we conclude that the functions $\xi_{i}-G_{i}(x), i=1, \ldots \bar{n}$, are Casimirs of (47) for $e=0, e_{C}=0$, if and only if they are Casimirs for both the internal interconnection structure $J_{c l}(x, \xi)$ as well as for the dissipation structure $R_{c l}(x, \xi)$. Hence, as in (38), it follows directly how the closed-loop port-controlled Hamiltonian system with dissipation (47) for $e=0, e_{C}=0$ reduces to a system any multi-level set $\left\{(x, \xi) \mid \xi_{i}=G_{i}(x)+c_{i}, i=1, \ldots, \bar{n}\right\}$, by restricting both $J_{c l}$ and $R_{c l}$ to this multi-level set.

Example 3.2. [56] Consider the "plant" system

$$
\begin{aligned}
& {\left[\begin{array}{c}
\dot{q} \\
\dot{p}
\end{array}\right]=\left[\begin{array}{cc}
0 & 1 \\
-1 & 0
\end{array}\right]\left[\begin{array}{c}
\frac{\partial H}{\partial q} \\
\frac{\partial H}{\partial p}
\end{array}\right]+\left[\begin{array}{l}
0 \\
1
\end{array}\right] u} \\
& y=\left[\begin{array}{ll}
0 & 1
\end{array}\right]\left[\begin{array}{c}
\frac{\partial H}{\partial q} \\
\frac{\partial H}{\partial p}
\end{array}\right]
\end{aligned}
$$


with $q$ the position and $p$ being the momentum of the mass $m$, in feedback interconnection $\left(u=-y_{C}+e, u_{C}=y\right)$ with the controller system (see Figure 4)

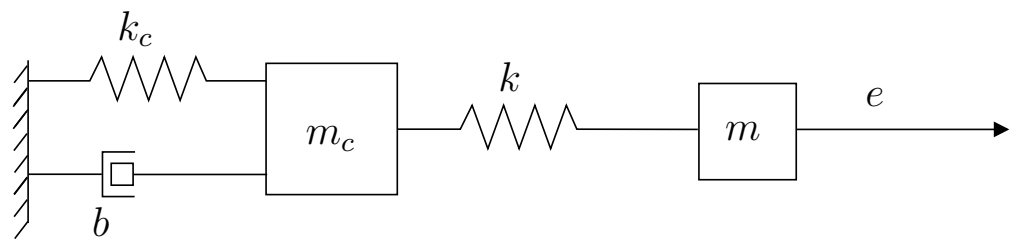

Figure 4: Controlled mass

$$
\begin{aligned}
& {\left[\begin{array}{c}
\dot{\Delta} q_{c} \\
\dot{p}_{c} \\
\dot{\Delta} q
\end{array}\right]=\left[\begin{array}{ccc}
0 & 1 & 0 \\
-1 & -b & 1 \\
0 & -1 & 0
\end{array}\right]\left[\begin{array}{l}
\frac{\partial H_{C}}{\partial \Delta q_{c}} \\
\frac{\partial H_{C}}{\partial p_{c}} \\
\frac{\partial H_{C}}{\partial \Delta q}
\end{array}\right]+\left[\begin{array}{l}
0 \\
0 \\
1
\end{array}\right] u_{C}} \\
& y_{C}=\frac{\partial H_{C}}{\partial \Delta q}
\end{aligned}
$$

where $\Delta q_{c}$ is the displacement of the spring $k_{c}, \Delta q$ is the displacement of the spring $k$, and $p_{c}$ is the momentum of the mass $m_{c}$. The plant Hamiltonian is $H(p)=\frac{1}{2 m} p^{2}$, and the controller Hamiltonian is given as $H_{C}\left(\Delta q_{c}, p_{c}, \Delta q\right)=\frac{1}{2}\left(\frac{p_{c}^{2}}{m_{c}}+k(\Delta q)^{2}+k_{c}\left(\Delta q_{c}\right)^{2}\right)$. The variable $b>0$ is the damping constant, and $e$ is an external force. The closed-loop system possesses the Casimir function

$$
C\left(q, \Delta q_{c}, \Delta q\right)=\Delta q-\left(q-\Delta q_{c}\right),
$$

implying that along the solutions of the closed-loop system

$$
\Delta q=q-\Delta q_{c}+c
$$

with $c$ a constant depending on the initial conditions. With the help of LaSalle's Invariance principle it can be shown that restricted to the invariant manifolds (54) the system is asymptotically stable for the equilibria $q=\Delta q_{c}=p=p_{c}=0$.

Let us next consider the special case $\bar{n}=n_{C}$, in which case we wish to relate all the controller state variables $\xi_{1}, \ldots, \xi_{n_{C}}$ to the plant state variables $x$ via Casimir functions $\xi_{1}-G_{1}(x), \ldots, \xi_{n_{C}}-G_{n_{C}}(x)$. Denoting $G=\left(G_{1}, \ldots, G_{n_{C}}\right)^{T}$ this means that $G$ should satisfy (see $(50))$

$$
\begin{aligned}
& \frac{\partial^{T} G}{\partial x}(x) J(x) \frac{\partial G}{\partial x}(x)=J_{C}(\xi) \\
& R(x) \frac{\partial G}{\partial x}(x)=0=R_{C}(\xi) \\
& \frac{\partial^{T} G}{\partial x}(x) J(x)=g_{C}(\xi) g^{T}(x)
\end{aligned}
$$

In this case the reduced dynamics on any multi-level set

$$
L_{C}=\left\{(x, \xi) \mid \xi_{i}=G_{i}(x)+c_{i}, i=1, \ldots n_{C}\right\}
$$


can be immediately recognized. Indeed, the $x$-coordinates also serve as coordinates for $L_{C}$. Furthermore, the $x$-dynamics of (47) with $e=0, e_{C}=0$ is given as

$$
\dot{x}=[J(x)-R(x)] \frac{\partial H}{\partial x}(x)-g(x) g_{C}^{T}(\xi) \frac{\partial H_{C}}{\partial \xi}(\xi) .
$$

Using the second and the third equality of (55) this can be rewritten as

$$
\dot{x}=[J(x)-R(x)]\left(\frac{\partial H}{\partial x}(x)+\frac{\partial G}{\partial x}(x) \frac{\partial H_{C}}{\partial \xi}(\xi)\right) .
$$

and by the chain-rule property for differentiation this reduces to the port-Hamiltonian system

$$
\dot{x}=[J(x)-R(x)] \frac{\partial H_{s}}{\partial x}(x),
$$

with the same interconnection and dissipation structure as before, but with shaped Hamiltonian $H_{s}$ given by

$$
H_{s}(x)=H(x)+H_{C}(G(x)+c) .
$$

An interpretation of the shaped Hamiltonian $H_{s}$ in terms of energy-balancing is the following. Since $R_{C}(\xi)=0$ by $(55)$ the controller Hamiltonian $H_{C}$ satisfies $\frac{d H_{C}}{d t}=u_{C}^{T} y_{C}$. Hence along any multi-level set $L_{C}$ given by (56), invariant for the closed loop port-Hamiltonian system (47) for $e=0, e_{C}=0$

$$
\frac{d H_{s}}{d t}=\frac{d H}{d t}+\frac{d H_{C}}{d t}=\frac{d H}{d t}-u^{T} y
$$

since $u=-y_{C}$ and $u_{C}=y$. Therefore, up to a constant,

$$
H_{s}(x(t))=H(x(t))-\int_{0}^{t} u^{T}(\tau) y(\tau) d \tau,
$$

and the shaped Hamiltonian $H_{s}$ is the original Hamiltonian $H$ minus the energy supplied to the plant system (44) by the controller system (45) (modulo a constant; depending on the initial states of the plant and controller).

Remark 3.3. Note that from a stability analysis point of view (62) can be regarded as an effective way of generating candidate Lyapunov functions $H_{s}$ from the Hamiltonian $H$. (Compare with the classical construction of Lur'e functions.)

Example 3.4. A mechanical system with damping and actuated by external forces $u \in \mathbb{R}^{m}$ is described as a port-Hamiltonian system

$$
\begin{aligned}
& {\left[\begin{array}{c}
\dot{q} \\
\dot{p}
\end{array}\right]=\left(\left[\begin{array}{cc}
0 & I_{k} \\
-I_{k} & 0
\end{array}\right]-\left[\begin{array}{cc}
0 & 0 \\
0 & D(q)
\end{array}\right]\right)\left[\begin{array}{c}
\frac{\partial H}{\partial q} \\
\frac{\partial H}{\partial p}
\end{array}\right]+\left[\begin{array}{c}
0 \\
B(q)
\end{array}\right] u} \\
& y=B^{T}(q) \frac{\partial H}{\partial p}
\end{aligned}
$$

with $x=\left[\begin{array}{l}q \\ p\end{array}\right]$, where $q \in \mathbb{R}^{k}$ are the generalized configuration coordinates, $p \in \mathbb{R}^{k}$ the generalized momenta, and $D(q)=D^{T}(q) \geq 0$ is the damping matrix. In most cases the Hamiltonian $H(q, p)$ takes the form

$$
H(q, p)=\frac{1}{2} p^{T} M^{-1}(q) p+P(q)
$$


where $M(q)=M^{T}(q)>0$ is the generalized inertia matrix, $\frac{1}{2} p^{T} M^{-1}(q) p=\frac{1}{2} \dot{q}^{T} M(q) \dot{q}$ is the kinetic energy, and $P(q)$ is the potential energy of the system. Now consider a general port-Hamiltonian controller system (45), with state space $\mathbb{R}^{m}$. Then the equations (55) for $G=\left(G_{1}(q, p), \ldots, G_{m}(q, p)\right)^{T}$ take the form

$$
\begin{gathered}
\frac{\partial^{T} G}{\partial q} \frac{\partial G}{\partial p}-\frac{\partial^{T} G}{\partial p} \frac{\partial G}{\partial q}=J_{C}(\xi) \\
D(q) \frac{\partial G}{\partial p}=0 \\
\frac{\partial^{T} G}{\partial p}=0, \frac{\partial^{T} G}{\partial q}=g_{C}(\xi) B^{T}(q)
\end{gathered}
$$

or equivalently

$$
J_{C}=0, \quad \frac{\partial G}{\partial p}=0, \quad g_{C}^{T}(\xi) B(q)=\frac{\partial G}{\partial q}(q)
$$

Now let $g_{C}(\xi)$ be the $m \times m$ identity matrix. Then there exists a solution $G=$ $\left(G_{1}(q), \ldots, G_{m}(q)\right)$ to $(66)$ if and only if the columns of the input force matrix $B(q)$ satisfy the integrability conditions

$$
\frac{\partial B_{i l}}{\partial q_{j}}(q)=\frac{\partial B_{j l}}{\partial q_{i}}(q), \quad i, j=1, \ldots k, \quad l=1, \ldots m
$$

Hence, if $B(q)$ satisfies (67), then the closed-loop port-Hamiltonian system (47) for the controller system (45) with $J_{C}=0$ admits Casimirs $\xi_{i}-G_{i}(q), i=1, \ldots, m$, leading to a reduced port-Hamiltonian system

$$
\begin{aligned}
& {\left[\begin{array}{c}
\dot{q} \\
\dot{p}
\end{array}\right]=\left(\left[\begin{array}{cc}
0 & I_{k} \\
-I_{k} & 0
\end{array}\right]-\left[\begin{array}{cc}
0 & 0 \\
0 & D(q)
\end{array}\right]\right)\left[\begin{array}{c}
\frac{\partial H_{s}}{\partial q} \\
\frac{\partial H_{s}}{\partial p}
\end{array}\right]+\left[\begin{array}{c}
0 \\
B(q)
\end{array}\right] e} \\
& y=B^{T}(q) \frac{\partial H_{s}}{\partial p}
\end{aligned}
$$

for the shaped Hamiltonian

$$
H_{s}(q, p)=H(q, p)+H_{C}\left(G_{1}(q)+c_{1}, \ldots, G_{m}(q)+c_{m}\right)
$$

If $H(q, p)$ is as given in $(64)$, then

$$
H_{s}(q, p)=\frac{1}{2} p^{T} M^{-1}(q) p+\left[P(q)+H_{C}\left(G_{1}(q)+c_{1}, \cdots, G_{m}(q)+c_{m}\right)\right]
$$

and the control amounts to shaping the potential energy of the system, see $[59,38]$.

\subsection{Passivity-based control of port-Hamiltonian systems}

In the previous section we have seen how under certain conditions the feedback interconnection of a port-Hamiltonian system having Hamiltonian $H$ (the "plant") with another portHamiltonian system with Hamiltonian $H_{C}$ (the "controller") leads to a reduced dynamics given by (see (59))

$$
\dot{x}=[J(x)-R(x)] \frac{\partial H_{s}}{\partial x}(x)
$$


for the shaped Hamiltonian $H_{s}(x)=H(x)+H_{C}(G(x)+c)$, with $G(x)$ a solution of (55). From a state feedback point of view the dynamics (71) could have been directly obtained by a state feedback $u=\alpha(x)$ such that

$$
g(x) \alpha(x)=[J(x)-R(x)] \frac{\partial H_{C}}{\partial x}(G(x)+c)
$$

Indeed, such an $\alpha(x)$ is given in explicit form as

$$
\alpha(x)=-g_{C}^{T}(G(x)+c) \frac{\partial H_{C}}{\partial \xi}(G(x)+c)
$$

A state feedback $u=\alpha(x)$ satisfying (72) is customarily called a passivity-based control law, since it is based on the passivity properties of the original plant system (44) and transforms (44) into another passive system with shaped storage function (in this case $H_{s}$ ).

Seen from this perspective we have shown in the previous section that the passivity-based state feedback $u=\alpha(x)$ satisfying (72) can be derived from the interconnection of the portHamiltonian plant system (44) with a port-Hamiltonian controller system (45). This fact has some favorable consequences. Indeed, it implies that the passivity-based control law defined by (72) can be equivalently generated as the feedback interconnection of the passive system (44) with another passive system (45). In particular, this implies an inherent invariance property of the controlled system: the plant system (71), the controller system (60), as well as any other passive system interconnected to (71) in a power-conserving fashion, may change in any way as long as they remain passive, and for any perturbation of this kind the controlled system will remain stable.

The implementation of the resulting passivity-based control $u=\alpha(x)$ is a somewhat complex issue. In cases of analog controller design the interconnection of the plant port-Hamiltonian system (44) with the port-Hamiltonian controller system (45) seems to be the logical option. Furthermore, in general it may be favorable to avoid an explicit state feedback, but instead to use the dynamic output feedback controller (45). On the other hand, in some applications the measurement of the passive output $y$ may pose some problems, while the state feedback $u=\alpha(x)$ is in fact easier to implement, as illustrated in the next example.

Example 3.5 (Example 3.4 continued). The passivity-based control $u=\alpha(x)$ resulting from (66) is given by (assuming $g_{C}$ to be the identity matrix and $B(q)$ to satisfy $(67)$ )

$$
u_{i}=-\frac{\partial H_{C}}{\partial \xi_{i}}(G(q)+c), \quad i=1, \ldots m
$$

This follows from (73), and can be directly checked by substituting (74) into (63) and using the equality $B(q)=\frac{\partial G}{\partial q}(q)$. Comparing the implementation of the state feedback controller (74) with the implementation of the port-Hamiltonian controller system based on the measurement of

$$
y=B^{T}(q) \frac{\partial H}{\partial p}(q, p)=B^{T}(q) \dot{q}
$$

one may note that the measurement of the generalized velocities (75) is in some cases (for example in a robotics context) more problematic than the measurement of the generalized positions (74). 
Remark 3.6. On the other hand, the control of the port-Hamiltonian plant system (44) by interconnection with the port-Hamiltonian controller system (45) allows for the possibility of inserting an asymptotically stabilizing damping not directly in the plant but instead in the controller system, cf. Example 3.2.

In the rest of this section we concentrate on the passivity-based (state feedback) control $u=\alpha(x)$. The purpose is to more systematically indicate how a port-Hamiltonian system with dissipation (44) may be asymptotically stabilized around a desired equilibrium $x^{*}$ in two steps:

I Shape by passivity-based control the Hamiltonian in such a way that it has a strict minimum at $x=x^{*}$. Then $x^{*}$ is a (marginally) stable equilibrium of the controlled system.

II Add damping to the system in such a way that $x^{*}$ becomes an asymptotically stable equilibrium of the controlled system.

As before, we shall concentrate on Step I. Therefore, let us consider a port-Hamiltonian system with dissipation (44) with $\mathcal{X}$ the $n$-dimensional state space manifold. Suppose we wish to stabilize the system around a desired equilibrium $x^{*}$, assigning a closed-loop energy function $H_{d}(x)$ to the system which has a strict minimum at $x^{*}$ (that is, $H_{d}(x)>H_{d}\left(x^{*}\right)$ for all $x \neq x^{*}$ in a neighbourhood of $\left.x^{*}\right)$. Denote

$$
H_{d}(x)=H(x)+H_{a}(x),
$$

where the to be defined function $H_{a}$ is the energy added to the system (by the control action). We have the following

Proposition 3.7. [39, 34] Assume we can find a feedback $u=\alpha(x)$ and a vector function $K(x)$ satisfying

$$
[J(x)-R(x)] K(x)=g(x) \alpha(x)
$$

such that

$$
\begin{aligned}
\text { (i) } \frac{\partial K_{i}}{\partial x_{j}}(x) & =\frac{\partial K_{j}}{\partial x_{i}}(x), \quad i, j=1, \ldots, n \\
\text { (ii) } K\left(x^{*}\right) & =-\frac{\partial H}{\partial x}\left(x^{*}\right) \\
\text { (iii) } \frac{\partial K}{\partial x}\left(x^{*}\right) & >-\frac{\partial^{2} H}{\partial x^{2}}\left(x^{*}\right)
\end{aligned}
$$

with $\frac{\partial K}{\partial x}$ the $n \times n$ matrix with $i$-th column given by $\frac{\partial K_{i}}{\partial x}(x)$, and $\frac{\partial^{2} H}{\partial x^{2}}\left(x^{*}\right)$ denoting the Hessian matrix of $H$ at $x^{*}$. Then the closed-loop system is a Hamiltonian system with dissipation

$$
\dot{x}=[J(x)-R(x)] \frac{\partial H_{d}}{\partial x}(x)
$$

where $H_{d}$ is given by (76), with $H_{a}$ such that

$$
K(x)=\frac{\partial H_{a}}{\partial x}(x)
$$

Furthermore, $x^{*}$ is a stable equilibrium of (79). 
A further generalization is to use state feedback in order to change the interconnection structure and the resistive structure of the plant system, and thereby to create more flexibility to shape the storage function for the (modified) port-controlled Hamiltonian system to a desired form. This methodology has been called Interconnection-Damping Assignment Passivity-Based Control (IDA-PBC) in [39, 40], and has been succesfully applied to a number of applications. The method is especially attractive if the newly assigned interconnection and resistive structures are judiciously chosen on the basis of physical considerations, and represent some "ideal" interconnection and resistive structures for the physical plant. For an extensive treatment of IDA-PBC we refer to [39, 40].

\section{Implicit port-Hamiltonian systems}

From a general modeling point of view physical systems are, at least in first instance, often described as DAE's, that is, a mixed set of differential and algebraic equations. This stems from the fact that in many modeling approaches the system under consideration is naturally regarded as obtained from interconnecting simpler sub-systems. These interconnections in general, give rise to algebraic constraints between the state space variables of the sub-systems; thus leading to implicit systems. While in the linear case one may argue that it is often relatively straightforward to eliminate the algebraic constraints, and thus to reduce the system to an explicit form, in the nonlinear case such a conversion from implicit to explicit form is usually fraught with difficulties. Indeed, if the algebraic constraints are nonlinear they need not be analytically solvable (locally or globally). More importantly perhaps, even if they are analytically solvable, then often one would prefer not to eliminate the algebraic constraints, because of the complicated and physically not easily interpretable expressions for the reduced system which may arise.

Therefore it is important to extend the framework of port-Hamiltonian systems, as sketched in the previous sections, to the context of implicit systems. In order to give the definition of an implicit port-Hamiltonian system (with dissipation) we first consider the notion of a Dirac structure, formalizing the concept of a power-conserving interconnection, and generalizing the notion of a structure matrix $J(x)$ as encountered before.

\subsection{Power-conserving interconnections}

Let us return to the basic setting of passivity, starting with a finite-dimensional linear space and its dual, in order to define power. Thus, let $\mathcal{F}$ be an $\ell$-dimensional linear space, and denote its dual (the space of linear functions on $\mathcal{F}$ ) by $\mathcal{F}^{*}$. The product space $\mathcal{F} \times \mathcal{F}^{*}$ is considered to be the space of power variables, with power defined by

$$
P=<f^{*} \mid f>, \quad\left(f, f^{*}\right) \in \mathcal{F} \times \mathcal{F}^{*},
$$

where $\left\langle f^{*}\right| f>$ denotes the duality product, that is, the linear function $f^{*} \in \mathcal{F}^{*}$ acting on $f \in \mathcal{F}$. Often we call $\mathcal{F}$ the space of flows $f$, and $\mathcal{F}^{*}$ the space of efforts e, with the power of an element $(f, e) \in \mathcal{F} \times \mathcal{F}^{*}$ denoted as $\langle e \mid f\rangle$.

Remark 4.1. If $\mathcal{F}$ is endowed with an inner product structure $<,>$, then $\mathcal{F}^{*}$ can be naturally identified with $\mathcal{F}$ in such a way that $\langle e \mid f\rangle=\langle e, f\rangle, f \in \mathcal{F}, e \in \mathcal{F}^{*} \simeq \mathcal{F}$. 
Example 4.2. Let $\mathcal{F}$ be the space of generalized velocities, and $\mathcal{F}^{*}$ be the space of generalized forces, then $\langle e \mid f\rangle$ is mechanical power. Similarly, let $\mathcal{F}$ be the space of currents, and $\mathcal{F}^{*}$ be the space of voltages, then $\langle e \mid f\rangle$ is electrical power.

There exists on $\mathcal{F} \times \mathcal{F}^{*}$ a canonically defined symmetric bilinear form

$$
<\left(f_{1}, e_{1}\right),\left(f_{2}, e_{2}\right)>_{\mathcal{F} \times \mathcal{F} *}:=<e_{1}\left|f_{2}>+<e_{2}\right| f_{1}>
$$

for $f_{i} \in \mathcal{F}, e_{i} \in \mathcal{F}^{*}, i=1,2$. Now consider a linear subspace

$$
S \subset \mathcal{F} \times \mathcal{F}^{*}
$$

and its orthogonal complement with respect to the bilinear form $<,>\mathcal{F} \times \mathcal{F}^{*}$ on $\mathcal{F} \times \mathcal{F}^{*}$, denoted as

$$
S^{\perp} \subset \mathcal{F} \times \mathcal{F}^{*}
$$

Clearly, if $S$ has dimension $d$, then the subspace $S^{\perp}$ has dimension $2 \ell-d$. (Since $\operatorname{dim}$ $\left(\mathcal{F} \times \mathcal{F}^{*}\right)=2 \ell$, and $<,>_{\mathcal{F} \times \mathcal{F}^{*}}$ is a non-degenerate form.)

Definition 4.3. $[9,12,11]$ A constant Dirac structure on $\mathcal{F}$ is a linear subspace $\mathcal{D} \subset \mathcal{F} \times \mathcal{F}^{*}$ such that

$$
\mathcal{D}=\mathcal{D}^{\perp}
$$

It immediately follows that the dimension of any Dirac structure $\mathcal{D}$ on an $\ell$-dimensional linear space is equal to $\ell$. Furthermore, let $(f, e) \in \mathcal{D}=\mathcal{D}^{\perp}$. Then by (82)

$$
0=<(f, e),(f, e)>_{\mathcal{F} \times \mathcal{F}^{*}}=2<e \mid f>.
$$

Thus for all $(f, e) \in \mathcal{D}$ we obtain

$$
<e \mid f>=0 .
$$

Hence a Dirac structure $\mathcal{D}$ on $\mathcal{F}$ defines a power-conserving relation between the power variables $(f, e) \in \mathcal{F} \times \mathcal{F}^{*}$.

Remark 4.4. The condition $\operatorname{dim} \mathcal{D}=\operatorname{dim} \mathcal{F}$ is intimately related to the usually expressed statement that a physical interconnection can not determine at the same time both the flow and effort (e.g. current and voltage, or velocity and force).

Constant Dirac structures admit different matrix representations. Here we just list a number of them, without giving proofs and algorithms to convert one representation into another, see e.g. [11].

Let $\mathcal{D} \subset \mathcal{F} \times \mathcal{F}^{*}$, with $\operatorname{dim} \mathcal{F}=\ell$, be a constant Dirac structure. Then $\mathcal{D}$ can be represented as

1. (Kernel and Image representation, $[11,50])$.

$$
\mathcal{D}=\left\{(f, e) \in \mathcal{F} \times \mathcal{F}^{*} \mid F f+E e=0\right\}
$$

for $\ell \times \ell$ matrices $F$ and $E$ satisfying

(i) $E F^{T}+F E^{T}=0$

(ii) $\operatorname{rank}[F: E]=\ell$ 
Equivalently,

$$
\mathcal{D}=\left\{(f, e) \in \mathcal{F} \times \mathcal{F}^{*} \mid f=E^{T} \lambda, e=F^{T} \lambda, \lambda \in \mathbb{R}^{\ell}\right\}
$$

2. (Constrained input-output representation, [11]).

$$
\mathcal{D}=\left\{(f, e) \in \mathcal{F} \times \mathcal{F}^{*} \mid f=J e+G \lambda, G^{T} e=0\right\}
$$

for an $\ell \times \ell$ skew-symmetric matrix $J$, and a matrix $G$ such that $\operatorname{Im} G=$ $\{f \mid(f, 0) \in \mathcal{D}\}$. Furthermore, $\operatorname{Ker} J=\{e \mid(0, e) \in \mathcal{D}\}$.

3. (Hybrid input-output representation, [6]).

Let $\mathcal{D}$ be given as in (88). Suppose rank $F=\ell^{1}(\leq \ell)$. Select $\ell^{1}$ independent columns of $F$, and group them into a matrix $F^{1}$. Write (possibly after permutations) $F=\left[F^{1}: F^{2}\right]$ and, correspondingly $E=\left[E^{1}: E^{2}\right], f=\left[\begin{array}{l}f^{1} \\ f^{2}\end{array}\right], e=\left[\begin{array}{c}e^{1} \\ e^{2}\end{array}\right]$. Then the matrix $\left[F^{1} \vdots E^{2}\right]$ can be shown to be invertible, and

$$
\mathcal{D}=\left\{\left(\begin{array}{l}
f^{1} \\
f^{2}
\end{array}\right),\left(\begin{array}{l}
e^{1} \\
e^{2}
\end{array}\right) \mid\left(\begin{array}{l}
f^{1} \\
e^{2}
\end{array}\right)=J\left(\begin{array}{l}
e^{1} \\
f^{2}
\end{array}\right)\right\}
$$

with $J:=-\left[F^{1}: E^{2}\right]^{-1}\left[F^{2}: E^{1}\right]$ skew-symmetric.

4. (Canonical coordinate representation, [9]).

There exist linear coordinates $(q, p, r, s)$ for $\mathcal{F}$ such in these coordinates and dual coordinates for $\mathcal{F}^{*},(f, e)=\left(f_{q}, f_{p}, f_{r}, f_{s}, e_{q}, e_{p}, e_{r}, e_{s}\right) \in \mathcal{D}$ if and only if

$$
\left\{\begin{array}{l}
f_{q}=e_{p}, \quad f_{p}=-e_{q} \\
f_{r}=0, \quad e_{s}=0
\end{array}\right.
$$

Example 4.5. Kirchhoff's laws are an example of (88), taking $\mathcal{F}$ the space of currents and $\mathcal{F}^{*}$ the space of voltages.

Given a Dirac structure $\mathcal{D}$ on $\mathcal{F}$, the following subspaces of $\mathcal{F}$, respectively $\mathcal{F}^{*}$, are of importance

$$
\begin{aligned}
G_{1} & :=\left\{f \in \mathcal{F} \mid \exists e \in \mathcal{F}^{*} \text { s.t. }(f, e) \in \mathcal{D}\right\} \\
P_{1} & :=\left\{e \in \mathcal{F}^{*} \mid \exists f \in \mathcal{F} \text { s.t. }(f, e) \in \mathcal{D}\right\}
\end{aligned}
$$

The subspace $G_{1}$ expresses the set of admissible flows, and $P_{1}$ the set of admissible efforts. It follows from the image representation (90) that

$$
\begin{aligned}
G_{1} & =\operatorname{Im} E^{T} \\
P_{1} & =\operatorname{Im} F^{T}
\end{aligned}
$$




\subsection{Implicit port-Hamiltonian systems}

From a network modeling perspective a (lumped-parameter) physical system is naturally described by a set of (possibly multi-dimensional) energy-storing elements, a set of energydissipating or resistive elements, and a set of ports (by which interaction with the environment can take place), interconnected to each other by a power-conserving interconnection, see Figure 5 .

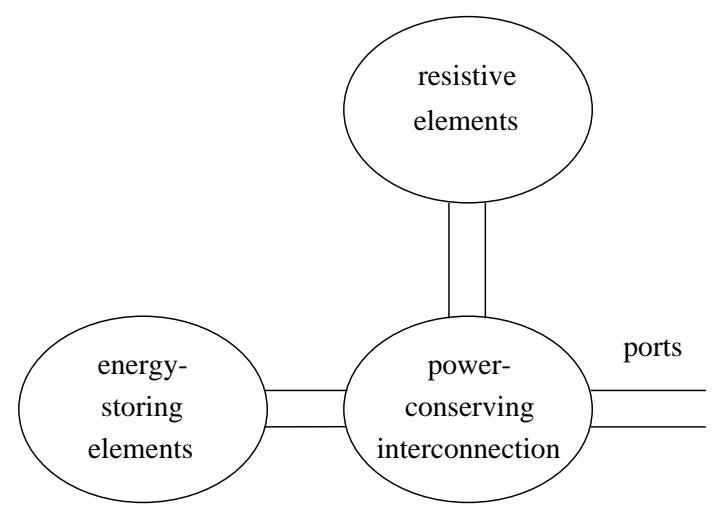

Figure 5: Implicit port-Hamiltonian system with dissipation

Here the power-conserving interconnection also includes power-conserving elements like (in the electrical domain) transformers, gyrators, or (in the mechanical domain) transformers, kinematic pairs and kinematic constraints.

Associated with the energy-storing elements are energy-variables $x_{1}, \cdots, x_{n}$, being coordinates for some $n$-dimensional state space manifold $\mathcal{X}$, and a total energy $H: \mathcal{X} \rightarrow \mathbb{R}$. The powerconserving interconnection is formalized in first instance (see later on for the non-constant case) by a constant Dirac structure $\mathcal{D}$ on the finite-dimensional linear space $\mathcal{F}:=\mathcal{F}_{S} \times \mathcal{F}_{R} \times \mathcal{F}_{P}$, with $\mathcal{F}_{S}$ denoting the space of flows $f_{S}$ connected to the energy-storing elements, $\mathcal{F}_{R}$ denoting the space of flows $f_{R}$ connected to the dissipative (resistive) elements, and $\mathcal{F}_{P}$ the space of external flows $f_{P}$ which can be connected to the environment. Dually, we write $\mathcal{F}^{*}=$ $\mathcal{F}_{S}^{*} \times \mathcal{F}_{R}^{*} \times \mathcal{F}_{P}^{*}$, with $e_{S} \in \mathcal{F}_{S}^{*}$ the efforts connected to the energy-storing elements, $e_{R} \in \mathcal{F}_{R}^{*}$ the efforts connected to the resistive elements, and $e_{P} \in \mathcal{F}_{P}^{*}$ the efforts to be connected to the environment of the system.

The flow variables of the energy-storing elements are given as $\dot{x}(t)=\frac{d x}{d t}(t), t \in \mathbb{R}$, and the effort variables of the energy-storing elements as $\frac{\partial H}{\partial x}(x(t))$ (implying that $\left\langle\frac{\partial H}{\partial x}(x(t)) \mid \dot{x}(t)\right\rangle=$ $\frac{d H}{d t}(x(t))$ is the increase in energy). In order to have a consistent sign convention for energy flow we put

$$
\begin{aligned}
f_{S} & =-\dot{x} \\
e_{S} & =\frac{\partial H}{\partial x}(x)
\end{aligned}
$$

Similarly, restricting to linear resistive elements as in (32), the flow and effort variables connected to the resistive elements are related as

$$
f_{R}=-R e_{R}
$$


for some matrix $R=R^{T} \geq 0$.

Substitution of (96) and (97) into the Dirac structure $\mathcal{D}$ leads to the following geometric description of the dynamics

$$
\left(f_{S}=-\dot{x}, f_{R}=-R e_{R}, f_{P}, e_{S}=\frac{\partial H}{\partial x}(x), e_{R}, e_{P}\right) \in \mathcal{D}
$$

We call (98) an implicit port-Hamiltonian system (with dissipation), defined with respect to the constant Dirac structure $\mathcal{D}$, the Hamiltonian $H$ and the resistive structure $R$.

An equational representation of an implicit port-Hamiltonian system is obtained by taking a matrix representation of the Dirac structure $\mathcal{D}$ as discussed in the previous subsection. For example, in kernel representation the Dirac structure on $\mathcal{F}=\mathcal{F}_{S} \times \mathcal{F}_{R} \times \mathcal{F}_{P}$ may be given as

$$
\begin{aligned}
\mathcal{D}= & \left\{\left(f_{S}, f_{R}, f_{P}, e_{S}, e_{R}, e_{P}\right) \mid\right. \\
& \left.F_{S} f_{S}+E_{S} e_{S}+F_{R} f_{R}+E_{R} e_{R}+F_{P} f_{P}+E_{P} e_{P}=0\right\}
\end{aligned}
$$

for certain matrices $F_{S}, E_{S}, F_{R}, E_{R}, F_{P}, E_{P}$ satisfying

$$
\begin{aligned}
& \text { (i) } E_{S} F_{S}^{T}+F_{S} E_{S}^{T}+E_{R} F_{R}^{T}+F_{R} E_{R}^{T}+E_{P} F_{P}^{T}+F_{P} E_{P}^{T}=0 \\
& \text { (ii) } \operatorname{rank}\left[F_{S} \vdots F_{R} \vdots F_{P} \vdots E_{S} \vdots E_{R} \vdots E_{P}\right]=\operatorname{dim} \mathcal{F}
\end{aligned}
$$

Then substitution of (96) and (97) into (99) yields the following set of differential-algebraic equations for the implicit port-Hamiltonian system

$$
F_{S} \dot{x}(t)=E_{S} \frac{\partial H}{\partial x}(x(t))-F_{R} R e_{R}+E_{R} e_{R}+F_{P} f_{P}+E_{P} e_{P}
$$

Different representations of the Dirac structure $\mathcal{D}$ lead to different representations of the implicit port-Hamiltonian system, and this freedom may be exploited for simulation and analysis.

Actually, for many purposes this definition of port-Hamiltonian system is not general enough, since often the Dirac structure is not constant, but modulated by the state variables $x$. In this case the matrices $F_{S}, E_{S}, F_{R}, E_{R}, F_{P}, E_{P}$ in the kernel representation depend (smoothly) on $x$, leading to the implicit port-Hamiltonian system

$$
\begin{aligned}
& F_{S}(x(t)) \dot{x}(t)=E_{S}(x(t)) \frac{\partial H}{\partial x}(x(t))-F_{R}(x(t)) R e_{R}(t) \\
& +E_{R}(x(t)) e_{R}(t)+F_{P}(x(t)) f_{P}(t)+E_{P}(x(t)) e_{P}(t), \quad t \in \mathbb{R}
\end{aligned}
$$

with

$$
\begin{aligned}
& E_{S}(x) F_{S}^{T}(x)+F_{S}(x) E_{S}^{T}(x)+E_{R}(x) F_{R}^{T}(x)+F_{R}(x) E_{R}^{T}(x) \\
& +E_{P}(x) F_{P}^{T}(x)+F_{P}(x) E_{P}^{T}(x)=0, \quad \forall x \in \mathcal{X} \\
& \operatorname{rank}\left[F_{S}(x) \vdots F_{R}(x) \vdots F_{P}(x) \vdots E_{S}(x) \vdots E_{R}(x) \vdots E_{P}(x)\right]=\operatorname{dim} \mathcal{F}
\end{aligned}
$$


Remark 4.6. Strictly speaking the flow and effort variables $\dot{x}(t)=-f_{S}(t)$, respectively $\frac{\partial H}{\partial x}(x(t))=e_{S}(t)$, are not living in a constant linear space $\mathcal{F}_{S}$, respectively $\mathcal{F}_{S}^{*}$, but instead in the tangent spaces $T_{x(t)} \mathcal{X}$, respectively co-tangent spaces $T_{x(t)}^{*} \mathcal{X}$, to the state space manifold $\mathcal{X}$. This is formalized in the definition of a non-constant Dirac structure on a manifold; see the references $[9,12,11,47]$.

It can be checked that the definition of a port-Hamiltonian system as given in (33) is a special case of (102), see [47]. By the power-conservation property of a Dirac structure (cf. (87)) it follows directly that any implicit port-Hamiltonian system satisfies the energy-balance

$$
\begin{aligned}
\frac{d H}{d t}(x(t)) & =<\frac{\partial H}{\partial x}(x(t)) \mid \dot{x}(t)>= \\
& =-e_{R}^{T}(t) \operatorname{Re}_{R}(t)+e_{P}^{T}(t) f_{P}(t),
\end{aligned}
$$

as was the case for an (explicit) port-Hamiltonian system (33).

The algebraic constraints that are present in the implicit system (102) are expressed by the subspace $P_{1}$, and the Hamiltonian $H$. In fact, since the Dirac structure $\mathcal{D}$ is modulated by the $x$-variables, also the subspace $P_{1}$ is modulated by the $x$-variables, and thus the effort variables $e_{S}, e_{R}$ and $e_{P}$ necessarily satisfy

$$
\left(e_{S}, e_{R}, e_{P}\right) \in P_{1}(x), \quad x \in \mathcal{X}
$$

or, because of (95),

$$
e_{S} \in \operatorname{Im} F_{S}^{T}(x), e_{R} \in \operatorname{Im} F_{R}^{T}(x), e_{P} \in \operatorname{Im} F_{P}^{T}(x) .
$$

The second and third inclusions entail the expression of $e_{R}$ and $e_{P}$ in terms of the other variables, while the first inclusion determines, since $e_{S}=\frac{\partial H}{\partial x}(x)$, the following algebraic constraints on the state variables

$$
\frac{\partial H}{\partial x}(x) \in \operatorname{Im} F_{S}^{T}(x) .
$$

Remark 4.7. Under certain non-degeneracy conditions the elimination of the algebraic constraints (107) for an implicit port-Hamiltonian system (98) can be shown to result in an explicit port-Hamiltonian system.

The Casimir functions $C: \mathcal{X} \rightarrow \mathbb{R}$ of the implicit system (102) are determined by the subspace $G_{1}(x)$. Indeed, necessarily $\left(f_{S}, f_{R}, f_{P}\right) \in G_{1}(x)$, and thus by (95)

$$
f_{S} \in \operatorname{Im} E_{S}^{T}(x), f_{R} \in \operatorname{Im} E_{R}^{T}(x), f_{P} \in \operatorname{Im} E_{P}^{T}(x) .
$$

Since $f_{S}=\dot{x}(t)$, the first inclusion yields the flow constraints

$$
\dot{x}(t) \in \operatorname{Im} E_{S}^{T}(x(t)), \quad t \in \mathbb{R} .
$$

Thus $C: \mathcal{X} \rightarrow \mathbb{R}$ is a Casimir function if $\frac{d C}{d t}(x(t))=\frac{\partial^{T} C}{\partial x}(x(t)) \dot{x}(t)=0$ for all $\dot{x}(t) \in$ $\operatorname{Im} E_{S}^{T}(x(t))$. Hence $C: \mathcal{X} \rightarrow \mathbb{R}$ is a Casimir of the implicit port-Hamiltonian system (98) if it satisfies the set of p.d.e.'s

$$
\frac{\partial C}{\partial x}(x) \in \operatorname{Ker} E_{S}(x)
$$


Remark 4.8. Note that $C: \mathcal{X} \rightarrow \mathbb{R}$ satisfying (110) is a Casimir function of (98) in a strong sense: it is a dynamical invariant $\left(\frac{d C}{d t}(x(t))=0\right)$ for every port behavior and every resistive relation (97).

Example 4.9. [11, 51] The constrained Hamiltonian equations (22) can be viewed as an implicit port-Hamiltonian system, with respect to the Dirac structure $\mathcal{D}$, given in constrained input-output representation (91) by

$$
\begin{aligned}
& \mathcal{D}=\left\{\left(f_{S}, f_{P}, e_{S}, e_{P}\right) \mid 0=A^{T}(q) e_{S}, e_{P}=B^{T}(q) e_{S},\right. \\
& \left.-f_{S}=\left[\begin{array}{cc}
0 & I_{k} \\
-I_{k} & 0
\end{array}\right] e_{S}+\left[\begin{array}{c}
0 \\
A(q)
\end{array}\right] \lambda+\left[\begin{array}{c}
0 \\
B(q)
\end{array}\right] f_{P}, \lambda \in \mathbb{R}^{r}\right\}
\end{aligned}
$$

In this case, the algebraic constraints on the state variables $(q, p)$ are given as

$$
0=A^{T}(q) \frac{\partial H}{\partial p}(q, p)
$$

while the Casimir functions $C$ are determined by the equations

$$
\frac{\partial^{T} C}{\partial q}(q) \dot{q}=0, \quad \text { for all } \dot{q} \text { satisfying } A^{T}(q) \dot{q}=0 .
$$

Hence, finding Casimir functions amounts to integrating the kinematic constraints $A^{T}(q) \dot{q}=$ 0. In particular, if the kinematic constraints are holonomic, and thus can be expressed as (19), then $\bar{q}_{k-r+1}, \cdots, \bar{q}_{k}$ generate all the Casimir functions.

Remark 4.10. For a proper notion of integrability of non-constant Dirac structures, generalizing the integrability conditions (12) of the structure matrix $J(x)$, we refer e.g. to [11].

In principle, the theory presented before for explicit port-Hamiltonian systems can be directly extended, mutatis mutandis, to implicit port-Hamiltonian system. In particular, the standard feedback interconnection of an implicit port-Hamiltonian system $P$ with port variables $f_{P}, e_{P}$

(the "plant") with another implicit port-Hamiltonian system with port variables $f_{P}^{C}, e_{P}^{C}$ (the "controller") is readily seen to result in a closed-loop implicit port-Hamiltonian system with port variables. Furthermore, as in the explicit case, the Hamiltonian of this closed-loop system is just the sum of the Hamiltonian of the plant port-Hamiltonian system and the Hamiltonian of the controller port-Hamiltonian system. Finally, the Casimir analysis for the closed-loop system can be performed along the same lines as before.

\section{Distributed-parameter port-Hamiltonian systems}

From a modeling and control point of view it is very desirable to be able to include distributedparameter components into the Hamiltonian description of complex physical systems. However, in extending the Hamiltonian theory as for instance exposed in [37] to distributedparameter control systems a fundamental difficulty arises in the treatment of boundary conditions. Indeed, the treatment of infinite-dimensional Hamiltonian systems in the literature seems mostly focussed on systems with infinite spatial domain, where the variables go to zero for the spatial variables tending to infinity, or on systems with boundary conditions such that the energy exchange through the boundary is zero. On the other hand, from a control and 
interconnection point of view it is essential to be able to describe a distributed-parameter system with varying boundary conditions inducing energy exchange through the boundary, since in many applications interaction with the environment (e.g. actuation or measurement) takes place through the boundary of the system. Clear examples are the telegraph equations (describing the dynamics of a transmission line), where the boundary of the system is described by the behavior of the voltages and currents at both ends of the transmission line, or a vibrating string (or, more generally, a flexible beam), where it is natural to consider the evolution of the forces and velocities at the ends of the string. Furthermore, in both examples it is obvious that in general the boundary exchange of power (voltage times current in the transmission line example, and force times velocity for the vibrating string) will be non-zero, and that in fact one would like to consider the voltages and currents or forces and velocities as additional boundary variables of the system, which can be interconnected to other systems. Also for numerical integration and simulation of complex distibuted-parameter systems it is essential to be able to describe the complex system as the interconnection or coupling of its subsystems via their boundary variables; for example in the case of coupled fluid-solid dynamics.

From a mathematical point of view, it is not obvious how to incorporate non-zero energy flow through the boundary in the existing Hamiltonian framework for distributed-parameter systems. The problem is already illustrated by the Hamiltonian formulation of e.g. the Korteweg-de Vries equation (see e.g. [37]). Here for zero boundary conditions a Poisson bracket can be formulated with the use of the differential operator $\frac{d}{d x}$, since by integration by parts this operator is obviously skew-symmetric. However, for boundary conditions corresponding to non-zero energy flow the differential operator is not skew-symmetric anymore (since after integrating by parts the remainders are not zero ).

In $[54,28,29]$ we have provided a framework to overcome this fundamental problem by using the notion of an infinite-dimensional Dirac structure. The infinite-dimensional Dirac structure employed in these papers has a specific form by being defined on certain spaces of differential forms on the spatial domain of the system and its boundary, and making use of Stokes' theorem. Its construction emphasizes the geometrical content of the physical variables involved, by identifying them as differential $k$-forms, for appropriate $k$. This framework has been used [54] for a port-Hamiltonian representation of the telegrapher's equations of an ideal transmission line, Maxwell's equations on a bounded domain with non-zero Poynting vector at its boundary, a vibrating string with traction forces at its ends, and planar beam models $[17,18]$. Furthermore the framework has been extended to cover Euler's equations for an ideal fluid on a domain with permeable boundary, see also [53].

Throughout, let $Z$ be an $n$-dimensional smooth manifold with smooth $(n-1)$-dimensional boundary $\partial Z$, representing the space of spatial variables.

Denote by $\Omega^{k}(Z), k=0,1, \cdots, n$, the space of exterior $k$-forms on $Z$, and by $\Omega^{k}(\partial Z), k=$ $0,1, \cdots, n-1$, the space of $\mathrm{k}$-forms on $\partial Z$. (Note that $\Omega^{0}(Z)$, respectively $\Omega^{0}(\partial Z)$, is the space of smooth functions on $Z$, respectively $\partial Z$.) Clearly, $\Omega^{k}(Z)$ and $\Omega^{k}(\partial Z)$ are (infinitedimensional) linear spaces (over $\mathbb{R}$ ). Furthermore, there is a natural pairing between $\Omega^{k}(Z)$ and $\Omega^{n-k}(Z)$ given by

$$
<\beta \mid \alpha>:=\int_{Z} \beta \wedge \alpha \quad(\in \mathbb{R})
$$

with $\alpha \in \Omega^{k}(Z), \beta \in \Omega^{n-k}(Z)$, where $\wedge$ is the usual wedge product of differential forms yielding the $n$-form $\beta \wedge \alpha$. In fact, the pairing (114) is non-degenerate in the sense that if 
$<\beta \mid \alpha>=0$ for all $\alpha$, respectively for all $\beta$, then $\beta=0$, respectively $\alpha=0$.

Similarly, there is a pairing between $\Omega^{k}(\partial Z)$ and $\Omega^{n-1-k}(\partial Z)$ given by

$$
<\beta \mid \alpha>:=\int_{\partial Z} \beta \wedge \alpha
$$

with $\alpha \in \Omega^{k}(\partial Z), \beta \in \Omega^{n-1-k}(\partial Z)$. Now let us define the linear space

$$
\mathcal{F}_{p, q}:=\Omega^{p}(Z) \times \Omega^{q}(Z) \times \Omega^{n-p}(\partial Z),
$$

for any pair $p, q$ of positive integers satisfying

$$
p+q=n+1,
$$

and correspondingly let us define

$$
\mathcal{E}_{p, q}:=\Omega^{n-p}(Z) \times \Omega^{n-q}(Z) \times \Omega^{n-q}(\partial Z) .
$$

Then the pairing (114) and (115) yields a (non-degenerate) pairing between $\mathcal{F}_{p, q}$ and $\mathcal{E}_{p, q}$ (note that by (117) $(n-p)+(n-q)=n-1)$. As before, symmetrization of this pairing yields the following bilinear form on $\mathcal{F}_{p, q} \times \mathcal{E}_{p, q}$ with values in $\mathbb{R}$ :

$$
\begin{aligned}
& \ll\left(f_{p}^{1}, f_{q}^{1}, f_{b}^{1}, e_{p}^{1}, e_{q}^{1}, e_{b}^{1}\right),\left(f_{p}^{2}, f_{q}^{2}, f_{b}^{2}, e_{p}^{2}, e_{q}^{2}, e_{b}^{2}\right) \gg:= \\
& \int_{Z}\left[e_{p}^{1} \wedge f_{p}^{2}+e_{q}^{1} \wedge f_{q}^{2}+e_{p}^{2} \wedge f_{p}^{1}+e_{q}^{2} \wedge f_{q}^{1}\right]+\int_{\partial Z}\left[e_{b}^{1} \wedge f_{b}^{2}+e_{b}^{2} \wedge f_{b}^{1}\right]
\end{aligned}
$$

where for $i=1,2$

$$
\begin{aligned}
& f_{p}^{i} \in \Omega^{p}(Z), f_{q}^{i} \in \Omega^{q}(Z) \\
& e_{p}^{i} \in \Omega^{n-p}(Z), e_{p}^{i} \in \Omega^{n-q}(Z) \\
& f_{b}^{i} \in \Omega^{n-p}(\partial Z), e_{b}^{i} \in \Omega^{n-q}(\partial Z)
\end{aligned}
$$

The spaces of differential forms $\Omega^{p}(Z)$ and $\Omega^{q}(Z)$ will represent the energy variables of two different physical energy domains interacting with each other, while $\Omega^{n-p}(\partial Z)$ and $\Omega^{n-q}(\partial Z)$ will denote the boundary variables whose (wedge) product represents the boundary energy flow. For example, in Maxwell's equations (Example 5.4) we will have $n=3$ and $p=q=2$; with $\Omega^{p}(Z)=\Omega^{2}(Z)$, respectively $\Omega^{q}(Z)=\Omega^{2}(Z)$, being the space of electric field inductions, respectively magnetic field inductions, and $\Omega^{n-p}(\partial Z)=\Omega^{1}(\partial Z)$ denoting the electric and magnetic field intensities at the boundary, with product the Poynting vector.

Theorem 5.1. Consider $\mathcal{F}_{p, q}$ and $\mathcal{E}_{p, q}$ given in (116), (118) with $p, q$ satisfying (117), and bilinear form $\ll, \gg$ given by (119). Define the following linear subspace $D$ of $\mathcal{F}_{p, q} \times \mathcal{E}_{p, q}$

$$
\begin{aligned}
D= & \left\{\left(f_{p}, f_{q}, f_{b}, e_{p}, e_{q}, e_{b}\right) \in \mathcal{F}_{p, q} \times \mathcal{E}_{p, q} \mid\right. \\
& {\left[\begin{array}{c}
f_{p} \\
f_{q}
\end{array}\right]=\left[\begin{array}{cc}
0 & (-1)^{r} d \\
d & 0
\end{array}\right]\left[\begin{array}{l}
e_{p} \\
e_{q}
\end{array}\right], } \\
& {\left.\left[\begin{array}{c}
f_{b} \\
e_{b}
\end{array}\right]=\left[\begin{array}{cc}
1 & 0 \\
0 & -(-1)^{n-q}
\end{array}\right]\left[\begin{array}{l}
e_{p \mid \partial Z} \\
e_{q \mid \partial Z}
\end{array}\right]\right\} }
\end{aligned}
$$

where $\left.\right|_{\partial Z}$ denotes restriction to the boundary $\partial Z$, and $r:=p q+1$. Then $D=D^{\perp}$, that is, $D$ is a Dirac structure. 
The proof of Theorem 5.1 relies on Stokes' theorem, and the infinite-dimensional Dirac structure defined in Theorem 5.1 is therefore called a Stokes-Dirac structure.

The definition of a distributed-parameter Hamiltonian system with respect to a StokesDirac structure can now be stated as follows. Let $Z$ be an $n$-dimensional manifold with boundary $\partial Z$, and let $D$ be a Stokes-Dirac structure. Consider furthermore a Hamiltonian density (energy per volume element)

$$
\mathcal{H}: \Omega^{p}(Z) \times \Omega^{q}(Z) \times Z \rightarrow \Omega^{n}(Z)
$$

resulting in the total energy

$$
H:=\int_{Z} \mathcal{H} \in \mathbb{R}
$$

Recall, see (114), that there exists a non-degenerate pairing between $\Omega^{p}(Z)$ and $\Omega^{n-p}(Z)$, respectively between $\Omega^{q}(Z)$ and $\Omega^{n-q}(Z)$. This means that $\Omega^{n-p}(Z)$ and $\Omega^{n-q}(Z)$ can be regarded as dual spaces to $\Omega^{p}(Z)$, respectively $\Omega^{q}(Z)$ (although strictly contained in their functional analytic duals). Let now $\alpha_{p}, \partial \alpha_{p} \in \Omega^{p}(Z), \alpha_{q}, \partial \alpha_{q} \in \Omega^{q}(Z)$. Then under weak smoothness conditions on $\mathcal{H}$

$$
\begin{array}{r}
H\left(\alpha_{p}+\partial \alpha_{p}, \alpha_{q}+\partial \alpha_{q}\right)=\int_{Z} \mathcal{H}\left(\alpha_{p}+\partial \alpha_{p}, \alpha_{q}+\partial \alpha_{q}, z\right)= \\
\int_{Z} \mathcal{H}\left(\alpha_{p}, \alpha_{q}, z\right)+\int_{Z}\left[\delta_{p} H \wedge \partial \alpha_{p}+\delta_{q} H \wedge \partial \alpha_{q}\right]+\text { higher order terms in } \partial \alpha_{p}, \partial \alpha_{q}
\end{array}
$$

for certain differential forms

$$
\begin{aligned}
& \delta_{p} H \in \Omega^{n-p}(Z) \\
& \delta_{q} H \in \Omega^{n-q}(Z)
\end{aligned}
$$

Furthermore, from the non-degeneracity of the pairing between $\Omega^{p}(Z)$ and $\Omega^{n-p}(Z)$, respectively between $\Omega^{q}(Z)$ and $\Omega^{n-q}(Z)$, it immediately follows that these differential forms are uniquely determined. This means that $\left(\delta_{p} H, \delta_{q} H\right) \in \Omega^{n-p}(Z) \times \Omega^{n-q}(Z)$ can be regarded as the (partial) variational derivatives (see e.g. [37]) of $H$ at $\left(\alpha_{p}, \alpha_{q}\right) \in \Omega^{p}(Z) \times \Omega^{q}(Z)$. Throughout this paper we shall assume that the Hamiltonian $H$ admits variational derivatives satisfying (124).

Now consider a time-function

$$
\left(\alpha_{p}(t), \alpha_{q}(t)\right) \in \Omega^{p}(Z) \times \Omega^{q}(Z), \quad t \in \mathbb{R},
$$

and the Hamiltonian $H\left(\alpha_{p}(t), \alpha_{q}(t)\right)$ evaluated along this trajectory. It follows that at any time $t$

$$
\frac{d H}{d t}=\int_{Z}\left[\delta_{p} H \wedge \frac{\partial \alpha_{p}}{\partial t}+\delta_{q} H \wedge \frac{\partial \alpha_{q}}{\partial t}\right]
$$

The differential forms $\frac{\partial \alpha_{p}}{\partial t}, \frac{\partial \alpha_{q}}{\partial t}$ represent the generalized velocities of the energy variables $\alpha_{p}, \alpha_{q}$. They are connected to the Stokes-Dirac structure $D$ by setting

$$
\begin{aligned}
& f_{p}=-\frac{\partial \alpha_{p}}{\partial t} \\
& f_{q}=-\frac{\partial \alpha_{q}}{\partial t}
\end{aligned}
$$


(again the minus sign is included to have a consistent energy flow description). Since the right-hand side of (127) is the rate of increase of the stored energy $H$, we set

$$
\begin{aligned}
& e_{p}=\delta_{p} H \\
& e_{q}=\delta_{q} H
\end{aligned}
$$

Definition 5.2. The distributed-parameter port-Hamiltonian system with $n$-dimensional manifold of spatial variables $Z$, state space $\Omega^{p}(Z) \times \Omega^{q}(Z)$ (with $p+q=n+1$ ), StokesDirac structure $D$ given by (121), and Hamiltonian $H$, is given as (with $r=p q+1$ )

$$
\begin{aligned}
{\left[\begin{array}{c}
-\frac{\partial \alpha_{p}}{\partial t} \\
-\frac{\partial \alpha_{q}}{\partial t}
\end{array}\right] } & =\left[\begin{array}{cc}
0 & (-1)^{r} d \\
d & 0
\end{array}\right]\left[\begin{array}{c}
\delta_{p} H \\
\delta_{q} H
\end{array}\right] \\
{\left[\begin{array}{c}
f_{b} \\
e_{b}
\end{array}\right] } & =\left[\begin{array}{cc}
1 & 0 \\
0 & -(-1)^{n-q}
\end{array}\right]\left[\begin{array}{c}
\left.\delta_{p} H\right|_{\partial Z} \\
\left.\delta_{q} H\right|_{\partial Z}
\end{array}\right]
\end{aligned}
$$

By the power-conserving property of any Dirac structure it immediately follows that for any $\left(f_{p}, f_{q}, f_{b}, e_{p}, e_{q}, e_{b}\right)$ in the Stokes-Dirac structure $D$

$$
\int_{Z}\left[e_{p} \wedge f_{p}+e_{q} \wedge f_{q}\right]+\int_{\partial Z} e_{b} \wedge f_{b}=0
$$

Hence by substitution of (128), (129) and using (127) we obtain

Proposition 5.3. Consider the distributed parameter port-Hamiltonian system (130). Then

$$
\frac{d H}{d t}=\int_{\partial Z} e_{b} \wedge f_{b}
$$

expressing that the increase in energy on the domain $Z$ is equal to the power supplied to the system through the boundary $\partial Z$.

The system (130) can be called a (nonlinear) boundary control system in the sense of e.g. [14]. Indeed, we could interpret $f_{b}$ as the boundary control inputs to the system, and $e_{b}$ as the measured outputs (or the other way around).

Example 5.4 (Maxwell's equations). Let $Z \subset \mathbb{R}^{3}$ be a 3-dimensional manifold with boundary $\partial Z$, defining the spatial domain, and consider the electromagnetic field in $Z$. The energy variables are the electric field induction 2-form $\alpha_{p}=\mathcal{D} \in \Omega^{2}(Z)$ :

$$
\mathcal{D}=\frac{1}{2} D_{i j}(t, z) d z^{i} \wedge d z^{j}
$$

and the magnetic field induction 2 -form $\alpha_{q}=\mathcal{B} \in \Omega^{2}(Z)$ :

$$
\mathcal{B}=\frac{1}{2} B_{i j}(t, z) d z^{i} \wedge d z^{j}
$$

The corresponding Stokes-Dirac structure $(n=3, p=2, q=2)$ is given as (cf. (121))

$$
\left[\begin{array}{c}
f_{p} \\
f_{q}
\end{array}\right]=\left[\begin{array}{cc}
0 & -d \\
d & 0
\end{array}\right]\left[\begin{array}{l}
e_{p} \\
e_{q}
\end{array}\right],\left[\begin{array}{l}
f_{b} \\
e_{b}
\end{array}\right]=\left[\begin{array}{ll}
1 & 0 \\
0 & 1
\end{array}\right]\left[\begin{array}{c}
e_{p \mid \partial Z} \\
e_{q \mid \partial Z}
\end{array}\right]
$$


Usually in this case one does not start with the definition of the total energy (Hamiltonian) $H$, but instead with the co-energy variables $\delta_{p} H, \delta_{q} H$, given, respectively, as the electric field intensity $\mathcal{E} \in \Omega^{1}(Z)$ :

$$
\mathcal{E}=E_{i}(t, z) d z^{i}
$$

and the magnetic field intensity $\mathcal{H} \in \Omega^{1}(Z)$ :

$$
\mathcal{H}=H_{i}(t, z) d z^{i}
$$

They are related to the energy variables through the constitutive relations of the medium (or material equations)

$$
\begin{aligned}
& * \mathcal{D}=\epsilon \mathcal{E} \\
& * \mathcal{B}=\mu H
\end{aligned}
$$

with the scalar functions $\epsilon(t, z)$ and $\mu(t, z)$ denoting the electric permittivity, respectively magnetic permeability, and $*$ denoting the Hodge star operator (corresponding to a Riemannian metric on $Z$ ), converting 2 -forms into 1 -forms. Then one defines the Hamiltonian $H$ as

$$
H=\int_{Z} \frac{1}{2}(\mathcal{E} \wedge \mathcal{D}+\mathcal{H} \wedge \mathcal{B}),
$$

and one immediately verifies that $\delta_{p} H=\mathcal{E}, \delta_{q} H=\mathcal{H}$.

Nevertheless there are other cases (corresponding to a nonlinear theory of the electromagnetic field, such as the Born-Infeld theory, see e.g. [21]) where one starts with a more general Hamiltonian $H=\int_{Z} h$, with the energy density $h(\mathcal{D}, B)$ being a more general expression than $\frac{1}{2}\left(\epsilon^{-1} * \mathcal{D} \wedge \mathcal{D}+\mu^{-1} * \mathcal{B} \wedge \mathcal{B}\right)$.

Assuming that there is no current in the medium Maxwell's equations can now be written as (see $[21])$

$$
\begin{aligned}
& \frac{\partial \mathcal{D}}{\partial t}=d \mathcal{H} \\
& \frac{\partial \mathcal{B}}{\partial t}=-d \mathcal{E}
\end{aligned}
$$

Explicitly taking into account the behavior at the boundary, Maxwell's equations on a domain $Z \subset \mathbb{R}^{3}$ are then represented as the port-Hamiltonian system with respect to the Stokes-Dirac structure given by (135), as

$$
\begin{aligned}
{\left[\begin{array}{l}
-\frac{\partial \mathcal{D}}{\partial t} \\
-\frac{\partial B}{\partial t}
\end{array}\right] } & =\left[\begin{array}{cc}
0 & -d \\
d & 0
\end{array}\right]\left[\begin{array}{l}
\delta_{D} H \\
\delta_{B} H
\end{array}\right] \\
{\left[\begin{array}{l}
f_{b} \\
e_{b}
\end{array}\right] } & =\left[\begin{array}{l}
\left.\delta_{D} H\right|_{\partial Z} \\
\left.\delta_{B} H\right|_{\partial Z}
\end{array}\right]
\end{aligned}
$$

Note that the first line of (140) is nothing else than (the differential version of) Ampère's law, while the second line of (140) is Faraday's law. Hence the Stokes-Dirac structure in (140), (141) expresses the basic physical laws connecting $\mathcal{D}, \mathcal{B}, \mathcal{H}$ and $\mathcal{E}$.

The energy-balance (132) in the case of Maxwell's equations takes the form

$$
\frac{d H}{d t}=\int_{\partial Z} \delta_{B} H \wedge \delta_{D} H=\int_{\partial Z} \mathcal{H} \wedge \mathcal{E}=-\int_{\partial Z} \mathcal{E} \wedge \mathcal{H}
$$

with $\mathcal{E} \wedge \mathcal{H}$ a 2-form corresponding to the Poynting vector (see [21]). 
Example 5.5 (Vibrating string). Consider an elastic string subject to traction forces at its ends. The spatial variable $z$ belongs to the interval $Z=[0,1] \subset \mathbb{R}$. Let us denote by $u(t, z)$ the displacement of the string. The elastic potential energy is a function of the strain given by the 1 -form

$$
\alpha_{q}(t)=\epsilon(t, z) d z=\frac{\partial u}{\partial z}(t, z) d z
$$

The associated co-energy variable is the stress given by the 0-form

$$
\sigma=T * \alpha_{q}
$$

with $T$ the elasticity modulus and $*$ the Hodge star operator. Hence the potential energy is the quadratic function

$$
U\left(\alpha_{q}\right)=\int_{0}^{1} \sigma \alpha_{q}=\int_{0}^{1} T * \alpha_{q} \wedge \alpha_{q}=\int_{0}^{1} T\left(\frac{\partial u}{\partial z}\right)^{2} d z
$$

and $\sigma=\delta_{q} U$.

The kinetic energy $K$ is a function of the kinetic momentum defined as the 1-form

$$
\alpha_{p}(t)=p(t, z) d z
$$

given by the quadratic function

$$
K\left(\alpha_{p}\right)=\int_{0}^{1} \frac{p^{2}}{\mu} d z
$$

The associated co-energy variable is the velocity given by the 0 -form

$$
v=\frac{1}{\mu} * \alpha_{p}=\delta_{p} K
$$

In this case the Dirac structure is the Stokes-Dirac structure for $n=p=q=1$, with an opposite sign convention leading to the equations (with $H:=U+K$ )

$$
\begin{aligned}
{\left[\begin{array}{c}
-\frac{\partial \alpha_{p}}{\partial t} \\
-\frac{\partial \alpha_{q}}{\partial t}
\end{array}\right] } & =\left[\begin{array}{cc}
0 & -d \\
-d & 0
\end{array}\right]\left[\begin{array}{l}
\delta_{p} H \\
\delta_{q} H
\end{array}\right] \\
{\left[\begin{array}{l}
f_{b} \\
e_{b}
\end{array}\right] } & =\left[\begin{array}{ll}
1 & 0 \\
0 & 1
\end{array}\right]\left[\begin{array}{l}
\left.\delta_{p} H\right|_{\partial Z} \\
\left.\delta_{q} H\right|_{\partial Z}
\end{array}\right]
\end{aligned}
$$

or, in more down-to-earth notation

$$
\begin{aligned}
\frac{\partial p}{\partial t} & =\frac{\partial \sigma}{\partial z}=\frac{\partial}{\partial z}(T \epsilon) \\
\frac{\partial \epsilon}{\partial t} & =\frac{\partial v}{\partial z}=\frac{\partial}{\partial z}\left(\frac{1}{\mu} p\right) \\
f_{b} & =\left.v\right|_{\{0,1\}} \\
e_{b} & =\left.\sigma\right|_{\{0,1\}}
\end{aligned}
$$


with boundary variables the velocity and stress at the ends of the string. Of course, by substituting $\epsilon=\frac{\partial u}{\partial z}$ into the 2 nd equation of (150) one obtains $\frac{\partial}{\partial z}\left(\frac{\partial u}{\partial t}-\frac{p}{\mu}\right)=0$, implying that

$$
p=\mu \frac{\partial u}{\partial t}+\mu f(t)
$$

for some function $f$, which may be set to zero. Substitution of (151) into the first equation of (150) then yields the wave equation

$$
\mu \frac{\partial^{2} u}{\partial t^{2}}=\frac{\partial}{\partial z}\left(T \frac{\partial u}{\partial z}\right)
$$

This framework can be extended to general beam models, see [17, 18].

\section{Conclusions and future research}

The theory presented in this paper opens up the way for many analysis, simulation and control problems. Its potential for set-point regulation has already received some attention (see [33, 34, 39, 40,47]), while the extension to tracking problems is wide open. In this context we also like to refer to some recent work concerned with the shaping of the Lagrangian, see e.g. [5]. Also, the control of mechanical systems with nonholonomic kinematic constraints can be fruitfully approached from this point of view, see e.g. [15], as well as the modelling and control of multi-body systems, see [32, 27, 58]. The framework of port-Hamiltonian systems seems perfectly suited to theoretical investigations on the topic of impedance control; see already [56] for some initial results in this direction. Furthermore, the connection with multi-modal (hybrid) systems, corresponding to port-Hamiltonian systems with varying interconnection structure, needs further investigations. Some applications of the framework of distributedparameter port-Hamiltonian systems have been already reported in [53, 42]; see also [55] for related work on smart structures. Finally, current research is concerned with the spatial discretization of port-Hamiltonian distributed-parameter systems to finite-dimensional portHamiltonian systems [16], with direct applications towards simulation and control of such systems.

\section{References}

[1] R.A. Abraham \& J.E. Marsden, Foundations of Mechanics (2nd edition), Reading, MA: Benjamin/Cummings, 1978.

[2] V. I. Arnold, B.A. Khesin, Topological Methods in Hydrodynamics, Springer Verlag, Applied Mathematical Sciences 125, New York, 1998.

[3] G. Blankenstein, Implicit Hamiltonian Systems; Symmetry and Interconnection, PhD thesis, University of Twente, The Netherlands, November 2000.

[4] G. Blankenstein, A.J. van der Schaft, "Symmetry and reduction in implicit generalized Hamiltonian systems", Rep. Math. Phys., 47, pp. 57-100, 2001. 
[5] A. Bloch, N. Leonard \& J.E. Marsden, "Matching and stabilization by the method of controlled Lagrangians", in Proc. 37th IEEE Conf. on Decision and Control, Tampa, FL, pp. 1446-1451, 1998.

[6] A.M. Bloch \& P.E. Crouch, "Representations of Dirac structures on vector spaces and nonlinear $L C$ circuits", Proc. Symposia in Pure Mathematics, Differential Geometry and Control Theory, G. Ferreyra, R. Gardner, H. Hermes, H. Sussmann, eds., Vol. 64, pp. 103-117, AMS, 1999.

[7] P.C. Breedveld, Physical systems theory in terms of bond graphs, $\mathrm{PhD}$ thesis, University of Twente, Faculty of Electrical Engineering, 1984

[8] R.W. Brockett, "Control theory and analytical mechanics", in Geometric Control Theory, (eds. C. Martin, R. Hermann), Vol. VII of Lie Groups: History, Frontiers and Applications, Math. Sci. Press, Brookline, pp. 1-46, 1977.

[9] T.J. Courant, "Dirac manifolds", Trans. American Math. Soc., 319, pp. 631-661, 1990.

[10] P.E. Crouch \& A.J. van der Schaft, Variational and Hamiltonian Control Systems, Lect. Notes in Control and Inf. Sciences 101, Springer-Verlag, Berlin, 1987.

[11] M. Dalsmo \& A.J. van der Schaft, "On representations and integrability of mathematical structures in energy-conserving physical systems", SIAM J. Control and Optimization, 37, pp. 54-91, 1999.

[12] I. Dorfman, Dirac Structures and Integrability of Nonlinear Evolution Equations, John Wiley, Chichester, 1993.

[13] G. Escobar, A.J. van der Schaft \& R. Ortega, "A Hamiltonian viewpoint in the modelling of switching power converters", Automatica, Special Issue on Hybrid Systems, 35, pp. 445-452, 1999.

[14] H.O. Fattorini, "Boundary control systems", SIAM J. Control, 6, pp. 349-385, 1968.

[15] K. Fujimoto, T. Sugie, "Stabilization of a class of Hamiltonian systems with nonholonomic constraints via canonical transformations", Proc. European Control Conference '99, Karlsruhe, 31 August - 3 September 1999.

[16] G. Golo, V. Talasila, A.J. van der Schaft, "Approximation of the telegrapher's equations", Proc. 41st IEEE Conf. Decision and Control, Las Vegas, Nevada, December 2002.

[17] G. Golo, V. Talasila, A.J. van der Schaft, "A Hamiltonian formulation of the Timoshenko beam", Mechatronics 2002, pp. 838-847, Enschede, 24-26 June 2002.

[18] G. Golo, A.J. van der Schaft, S.Stramigioli, "Hamiltonian formulation of planar beams", Proceedings 2nd IFAC Workshop on Lagrangian and Hamiltonian Methods for Nonlinear Control, Eds. A. Astolfi, F. Gordillo, A.J. van der Schaft, pp. 169-174, Sevilla, 2003.

[19] G. Golo, A. van der Schaft, P.C. Breedveld, B.M. Maschke, "Hamiltonian formulation of bond graphs", Nonlinear and Hybrid Systems in Automotive Control Eds. R. Johansson, A. Rantzer, pp. 351-372, Springer London, 2003. 
[20] D.J. Hill \& P.J. Moylan, "Stability of nonlinear dissipative systems," IEEE Trans. Aut. Contr., AC-21, pp. 708-711, 1976.

[21] R.S. Ingarden, A. Jamiolkowski, Classical Electrodynamics, PWN-Polish Sc. Publ., Warszawa, Elsevier, 1985.

[22] A. Isidori, Nonlinear Control Systems (2nd Edition), Communications and Control Engineering Series, Springer-Verlag, London, 1989, 3rd Edition, 1995.

[23] J.E. Marsden \& T.S. Ratiu, Introduction to Mechanics and Symmetry, Texts in Applied Mathematics 17, Springer-Verlag, New York, 1994.

[24] B.M. Maschke \& A.J. van der Schaft, "Port-controlled Hamiltonian systems: Modelling origins and system-theoretic properties", in Proc. 2nd IFAC NOLCOS, Bordeaux, pp. 282-288, 1992.

[25] B.M. Maschke \& A.J. van der Schaft, "System-theoretic properties of port-controlled Hamiltonian systems", in Systems and Networks: Mathematical Theory and Applications, Vol. II, Akademie-Verlag, Berlin, pp. 349-352, 1994.

[26] B.M. Maschke \& A.J. van der Schaft, "Interconnection of systems: the network paradigm", in Proc. 35th IEEE Conf. on Decision and Control, Kobe, Japan, pp. 207-212, 1996.

[27] B.M. Maschke \& A.J. van der Schaft, "Interconnected Mechanical Systems, Part II: The Dynamics of Spatial Mechanical Networks", in Modelling and Control of Mechanical Systems, (eds. A. Astolfi, D.J.N. Limebeer, C. Melchiorri, A. Tornambe, R.B. Vinter), pp. 17-30, Imperial College Press, London, 1997.

[28] B.M. Maschke, A.J. van der Schaft, "Port controlled Hamiltonian representation of distributed parameter systems", Proc. IFAC Workshop on Lagrangian and Hamiltonian methods for nonlinear control, Princeton University, Editors N.E. Leonard, R. Ortega, pp.28-38, 2000.

[29] B.M. Maschke, A.J. van der Schaft, "Hamiltonian representation of distributed parameter systems with boundary energy flow", Nonlinear Control in the Year 2000. Eds. A. Isidori, F. Lamnabhi-Lagarrigue, W. Respondek, Lect. Notes Control and Inf. Sciences, vol. 258, Springer-Verlag, pp. 137-142, 2000.

[30] B.M. Maschke, A.J. van der Schaft \& P.C. Breedveld, "An intrinsic Hamiltonian formulation of network dynamics: non-standard Poisson structures and gyrators", J. Franklin Institute, vol. 329, no.5, pp. 923-966, 1992.

[31] B.M. Maschke, A.J. van der Schaft \& P.C. Breedveld, "An intrinsic Hamiltonian formulation of the dynamics of LC-circuits, IEEE Trans. Circ. and Syst., CAS-42, pp. 73-82, 1995.

[32] B.M. Maschke, C. Bidard \& A.J. van der Schaft, "Screw-vector bond graphs for the kinestatic and dynamic modeling of multibody systems", in Proc. ASME Int. Mech. Engg. Congress, 55-2, Chicago, U.S.A., pp. 637-644, 1994. 
[33] B.M. Maschke, R. Ortega \& A.J. van der Schaft, "Energy-based Lyapunov functions for forced Hamiltonian systems with dissipation", in Proc. 37th IEEE Conference on Decision and Control, Tampa, FL, pp. 3599-3604, 1998.

[34] B.M. Maschke, R. Ortega, A.J. van der Schaft \& G. Escobar, "An energy-based derivation of Lyapunov functions for forced systems with application to stabilizing control", in Proc. 14th IFAC World Congress, Beijing, Vol. E, pp. 409-414, 1999.

[35] J.I. Neimark \& N.A. Fufaev, Dynamics of Nonholonomic Systems, Vol. 33 of Translations of Mathematical Monographs, American Mathematical Society, Providence, Rhode Island, 1972.

[36] H. Nijmeijer \& A.J. van der Schaft, Nonlinear Dynamical Control Systems, SpringerVerlag, New York, 1990.

[37] P.J. Olver, Applications of Lie Groups to Differential Equations, Springer-Verlag, second edition, 1993.

[38] R. Ortega, A. Loria, P.J. Nicklasson \& H. Sira-Ramirez, Passivity-based Control of EulerLagrange Systems, Springer-Verlag, London, 1998.

[39] R. Ortega, A.J. van der Schaft, B.M. Maschke \& G. Escobar, "Interconnection and damping assignment passivity-based control of port-controlled Hamiltonian systems", Automatica, vol. 38, pp. 585-596, 2002.

[40] R. Ortega, A.J. van der Schaft, I. Mareels, \& B.M. Maschke, "Putting energy back in control", Control Systems Magazine, 21, pp. 18-33, 2001.

[41] H. M. Paynter, Analysis and design of engineering systems, M.I.T. Press, MA, 1960.

[42] H. Rodriguez, A.J. van der Schaft, R. Ortega, "On stabilization of nonlinear distributed parameter port-controlled Hamiltonian systems via energy-shaping", Proc. 40th IEEE Conf. on Decision and Control, Orlando, Florida, pp.131-136, 2001.

[43] A.J. van der Schaft, System theoretic properties of physical systems, CWI Tract 3, CWI, Amsterdam, 1984.

[44] A.J. van der Schaft, "Stabilization of Hamiltonian systems", Nonl. An. Th. Math. Appl., 10, pp. 1021-1035, 1986.

[45] A.J. van der Schaft, "Implicit Hamiltonian systems with symmetry",Rep. Math. Phys., 41, pp. 203-221, 1998.

[46] A.J. van der Schaft, "Interconnection and geometry", in The Mathematics of Systems and Control, From Intelligent Control to Behavioral Systems (eds. J.W. Polderman, H.L. Trentelman), Groningen, 1999.

[47] A.J. van der Schaft, $L_{2}$-Gain and Passivity Techniques in Nonlinear Control, 2nd revised and enlarged edition, Springer-Verlag, Springer Communications and Control Engineering series, p. xvi+249, London, 2000 (first edition Lect. Notes in Control and Inf. Sciences, vol. 218, Springer-Verlag, Berlin, 1996). 
[48] A.J. van der Schaft, M. Dalsmo \& B.M. Maschke, "Mathematical structures in the network representation of energy-conserving physical systems", in Proc. 35th IEEE Conf. on Decision and Control, Kobe, Japan, pp. 201-206, 1996.

[49] A.J. van der Schaft \& B.M. Maschke, "On the Hamiltonian formulation of nonholonomic mechanical systems", Rep. Math. Phys., 34, pp. 225-233, 1994.

[50] A.J. van der Schaft \& B.M. Maschke, "The Hamiltonian formulation of energy conserving physical systems with external ports", Archiv für Elektronik und Übertragungstechnik, 49, pp. 362-371, 1995.

[51] A.J. van der Schaft \& B.M. Maschke, "Mathematical modeling of constrained Hamiltonian systems", in Proc. 3rd IFAC NOLCOS '95, Tahoe City, CA, pp. 678-683, 1995.

[52] A.J. van der Schaft \& B.M. Maschke, "Interconnected Mechanica5a Systems, Part I: Geometry of Interconnection and implicit Hamiltonian Systems", in Modelling and Control of Mechanical Systems, (eds. A. Astolfi, D.J.N. Limebeer, C. Melchiorri, A. Tornambe, R.B. Vinter), pp. 1-15, Imperial College Press, London, 1997.

[53] A.J. van der Schaft, B.M. Maschke, "Fluid dynamical systems as Hamiltonian boundary control systems", Proc. 40th IEEE Conf. on Decision and Control, Orlando, Florida, pp.4497-4502, 2001.

[54] A.J. van der Schaft, B.M. Maschke "Hamiltonian representation of distributed parameter systems with boundary energy flow", Journal of Geometry and Physics, vol.42, pp.166194, 2002.

[55] K. Schlacher, A. Kugi, "Control of mechanical structures by piezoelectric actuators and sensors". In Stability and Stabilization of Nonlinear Systems, eds. D. Aeyels, F. Lamnabhi-Lagarrigue, A.J. van der Schaft, Lecture Notes in Control and Information Sciences, vol. 246, pp. 275-292, Springer-Verlag, London, 1999.

[56] S. Stramigioli, From Differentiable Manifolds to Interactive Robot Control, PhD Dissertation, University of Delft, Dec. 1998.

[57] S. Stramigioli, B.M. Maschke \& A.J. van der Schaft, "Passive output feedback and port interconnection", in Proc. 4th IFAC NOLCOS, Enschede, pp. 613-618, 1998.

[58] S. Stramigioli, B.M. Maschke, C. Bidard, "A Hamiltonian formulation of the dynamics of spatial mechanism using Lie groups and screw theory", to appear in Proc. Symposium Commemorating the Legacy, Work and Life of Sir R.S. Ball, J. Duffy and H. Lipkin organizers, July 9-11, 2000, University of Cambridge, Trinity College, Cambridge, U.K..

[59] M. Takegaki \& S. Arimoto, "A new feedback method for dynamic control of manipulators", Trans. ASME, J. Dyn. Systems, Meas. Control, 103, pp. 119-125, 1981.

[60] A. Weinstein, "The local structure of Poisson manifolds", J. Differential Geometry, 18, pp. 523-557, 1983.

[61] J.C. Willems, "Dissipative dynamical systems - Part I: General Theory", Archive for Rational Mechanics and Analysis, 45, pp. 321-351, 1972. 